\title{
Revue sur les méthodes de dosage de la lignine utilisées en alimentation animale
}

\author{
Sylvie GIGER \\ I.N.R.A., Laboratoire de la Chaire de Zootechnie \\ Institut National agronomique Paris-Grignon \\ 16, rue Claude-Bernard, F 75231 Paris Cedex 05
}

\section{Résumé}

La lignine est un composé pariétal de nature non glucidique dont la structure est complexe et variable selon les aliments. C'est un facteur de variation important de la valeur énergétique des aliments, puisqu'elle est indigestible et entrave l'utilisation digestive des glucides pariétaux.

De nombreuses méthodes d'analyse, principalement de nature chimique, ont été utilisées pour déterminer la teneur de ce constituant. Cependant, aucune d'entre elles ne permet d'obtenir une fraction analytiquement pure et qui n'ait subi de perte préalable. Cette carence méthodologique peut s'expliquer par la complexité et la diversité des fractions «dites ligneuses》.

Les comparaisons des méthodes d'estimation de la lignine dans le contexte de l'alimentation animale sont rares. Avec les données actuellement disponibles, aucune méthode d'analyse ne s'avère être supérieure aux autres et les méthodes les plus utilisées sont celles qui sont les plus simples à mettre en œuvre. Il semble nécessaire d'entreprendre des études complémentaires de comparaison, notamment pour les aliments concentrés et les sous-produits dont la composition chimique et l'origine botanique sont beaucoup plus variables que celles des fourrages.

Mots clés : lignine, revue, estimation, aliments, signification nutritionnelle.

\section{Introduction}

La lignine est un terme générique qui désigne un ensemble de substances de haut poids moléculaire, de nature non glucidique et de constitution encore mal connue. Il semble maintenant admis que ce polymère tridimensionnel provient de la copolymérisation de trois alcools phénylpropénoïques (alcools coumarylique, coniférylique et sinapylique) (Freudenberg, 1965 et Monties, 1980). A la suite de Sarkanen \& LuDw IG (1971), certains auteurs préfèrent utiliser le terme de "lignines » à la place de celui de «lignine " pour bien mettre en évidence l'hétérogénéité des substances que l'on appelle ainsi. Monties \& LAPIERRE (1981) distinguent trois grands types d'hétérogénéité : hétérogénéité de la composition monomérique, hétérogénéité dans la fréquence des liaisons et hétérogénéité de l'hétéropolymérisation.

Dès 1860, Henneberg et Stohmann ont observé que la partie indigestible de l'extractif non azoté (schéma de WEENDE) d'une ration de foin et de paille distribuée 
à des vaches avait une composition proche de celle décrite par Schulze en 1857 pour la lignine (FIScher, 1961 b). Quelques années plus tard, DiETRICH \& Konig (1871) ont fait une observation analogue sur la fraction cellulose brute de la ration. D'autres études dont celles de Csonka, Phillips \& Jones (1929) ont montré que la lignine n'est vraisemblablement pas, ou que très peu, digérée par les animaux supérieurs. De plus, sa présence dans la paroi végétale entrave la digestion des autres constituants pariétaux, car les bactéries ne peuvent dégrader les tissus lignifiés comme AKıN (1979) et Harbers et al. (1981) l'ont montré par microscopie électronique. Cette observation est probablement liée au fait que la lignine est associée aux hémicelluloses par des liaisons de covalence (Morrison, 1979). De ce fait, la nature et la fréquence des liaisons lignine-glucides pariétaux constituent des facteurs de variation prépondérants de la digestibilité de la fraction pariétale des aliments. L'importance de ces facteurs est d'autant plus grande sur la digestibilité de la matière organique, donc sur la valeur énergétique des aliments, qu'ils contiennent une proportion élevée de paroi végétale.

De ce fait, de nombreuses études ont été entreprises depuis une centaine d'années pour évaluer la teneur en lignine des aliments destinés aux animaux domestiques et en déduire des lois de prévision de leur C.U.D. comme le montre notamment la revue de Fischer (1961 b). Des méthodes de dosage de la lignine très différentes ont été utilisées à l'occasion de ces travaux.

Depuis une vingtaine d'années, les recherches de modèles de prévision de plus cn plus précis et universels de la valeur énergétique des aliments et la simplification progressive des méthodes d'estimation de la lignine ont sensiblement accru l'intérêt de pouvoir estimer la teneur en lignine des aliments par des méthodes susceptibles d'être utilisées en grande série par des laboratoires de contrôle.

Il semble donc opportun de réaliser un travail bibliographique faisant le point des méthodes et travaux publiés pour tenter d'en extraire des bases de choix d'une méthode d'estimation de la lignine qui soit applicable en grande série afin d'apprécier la valeur nutritive énergétique des différents aliments distribués aux animaux domestiques.

\section{Les méthodes d'estimation de la lignine utilisées en alimentation animale}

Dès 1883, Fremy a su définir la lignine en des termes qui permettent de comprendre lcs principes sur lesquels sont fondées les principales méthodes de dosage :

«C'est la substance qui forme la partie principale des vaisseaux. Elle accompagne presque toujours les corps cellulosiques, elle lie entre elles les fibres et les cellules; ses caractères chimiques sont nets...; elle est insoluble dans le réactif ammoniacocuivrique, même après action des acides, elle résiste pendant longtemps à l'acide sulfurique concentré ; elle est rapidement attaquée par le chlore, par les hypochlorites et par les oxydants tels que l'acide azotique, l'acide chromique et le permanganate de potasse. La vasculose produit, sous ces différentes influences, des acides résineux solubles dans les alcalis et peut être ainsi séparée des corps cellulosiques ".

Les méthodes d'estimation de la lignine utilisées en alimentation animale sont issues de celles pratiquées dans l'industrie papetière. Elles ont été conçues pour des échantillons de bois, c'est-à-dire des substrats riches en constituants fibreux et ligneux et pauvres en matières azotées, graisses ou glucides solubles qui sont des substances susceptibles d'interférer dans le dosage de la lignine. De ce fait, ces méthodes de dosage 
peuvent être, dans certains cas, mal adaptées aux fourrages, sous-produits et aliments concentrés, employés en alimentation animale dont les teneurs en constituants non pariélaux sont parfois élevées.

\section{A. Méthodes gravimétriques}

1. Méthodes basées sur une hydrolyse en milieu acide concentré

Ces méthodes, dites lignines de KLASON, utilisent la propriété de l'insolubilité de la lignine dans un milieu acide concentré qui hydrolyse et dissout les autres constituants pariétaux (cellulose et hémicelluloses). Elles ont pour objectif l'obtention d'un résidu uniquement ligneux et sont toutes plus ou moins construtites sur le même schéma qui a été pour la première fois décrit dans son intégralité par Ellis, MATRONE \& MAYNARD (1946) (fig. 1). Chacune des étapes a pour objectif, soit d'extraire des substances qui pourraient former des produits de condensation avec la lignine au moment de l'attaque par l'acide concentré, soit de précipiter des formes solubles de la lignine suivant lordre suivant :

1) extraction des glucides cytoplasmiques, cires, matières grasses et pigments ;

2) extraction des protéines;

3) extraction des produits hémicellulosiques ;

4) extraction des produits cellulosiques;

5) précipitation de la lignine restée en solution après l'hydrolyse principale par post-hydrolyse.

extraction pendant 4 heures

avec un mélange alcool (32 p. 100) - benzène (64 p 100)

extraction for 4 hours

in an alcohol (32 p. 100) - benzene (64 p. 100) mixture

digestion pepsique en milieu chlorhydrique

[1 p. 100 pepsine dans $0,1 \mathrm{NHCl}$ à 40 " $\mathrm{C}$ (pendant la nuit)]

pepsic digestion in hydrochloric acid

[1 p. 100 pepsin in $0.1 \mathrm{NHCl}$ at $40^{\circ} \mathrm{C}$ (overnight)]

extraction avec reflux dans H.SO $_{4} 5$ p. 100 pendant 1 heure

refluxing extraction in $5 \mathrm{p} .100 \mathrm{H}_{\mathrm{S}} \mathrm{SO}$, for 1 hour

digestion avec $\mathrm{H}_{2} \mathrm{SO}_{4} 72$ p. 100 pendant 2 heures à $20^{\circ} \mathrm{C}$

digestion with 72 p. $100 \mathrm{H}_{2} \mathrm{SO}_{4}$ for 2 hours at $20^{\circ} \mathrm{C}$

$\downarrow$

dilution dans $\mathrm{H}_{2} \mathrm{SO}_{4} 3$ p. 100 et reflux pendant 2 heures

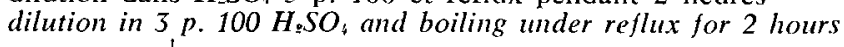

filtration du résidu

séchage et pesée

filtration of the residue

oven-drying and weighing

minéralisation

ashing

$\rightarrow \underset{\text { lignin }}{\text { lignine }}$

Fig. 1

Schéma de la méthode de Ellis, Matrone et Maynard (1946).

Method of Ellis, Matrone and Maynard (1946). 
Alors que les objectifs de chacune des étapes sont facilement discernables, les publications ne permettent pas toujours de cerner les motivations des choix techniques, ni même dans certains cas de juger objectivement de leur efficacité.

a) Extraction des glucides cytoplasmiques, cires, matières grasses, pigments, résines et tannins

Dans la méthode proposée par KLASON (1880), l'échantillon subit un prétraitement à l'alcool-benzène $(1: 2)$, suivi d'un lavage au mélange alcool-eau. De nombreuses méthodes comprennent un tel prétraitement dont la durée a varié de une (ARMITAGE, ASHWORTH \& FERguSON, 1948) à soixante heures (ShERRARd \& HARRIS, 1932) généralement effectué avec un appareil de type Soxhlet. Cette phase opératoire peut être complétée par un lavage avec de l'eau contenant de l'éther (McDougall \& DE LoNG, 1948) ou avec de l'alcool (Ellis, Matrone \& Maynard, 1946) afin d'éliminer les dernières traces de benzène.

A la suite des travaux de Bevenue \& Williams (1959) sur l'utilisation des détergents dans l'obtention des résidus pariétaux, ces prétraitements ont été supprimés et remplacés par des hydrolyses en milieu acide en présence de détergents : bromure et cétyltriméthylammonium (Van SoEst, 1963), fig. 2, laurylsulfate de sodium (FonNEsBECK, 1976, abeson K (benzène alkylsulfonate acide) (Nedoma, STAJGR \& MiKA, 1978) ou rodapon 80 p. 100 (benzène alkylsulfonate de sodium) (méthode de l'Oskar Kellner Institute : O.K.I.).

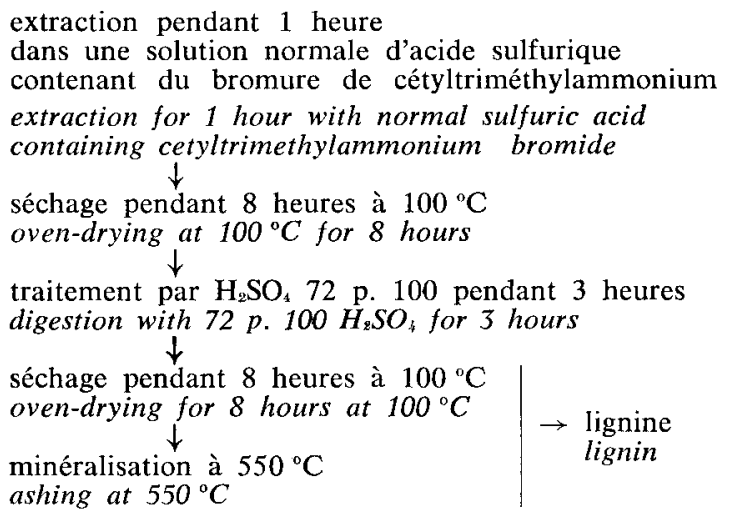

FIG. 2

Schéma de la méthode de Van Soest à l'acide sulfurique 72 p. 100 (Van Soest, 1963). Method of Van Soest with 72 p. $100 \mathrm{H}_{2} \mathrm{SO}_{4}$ (Van Soest, 1963).

\section{b) Extraction des protéines}

La méthode de déprotéinisation par la pepsine en milieu chlorhydrique $(0,1 \mathrm{~N})$ est celle qui a été la plus utilisée ( Crampton \& Maynard, 1938 ; Ellis, Matrone 


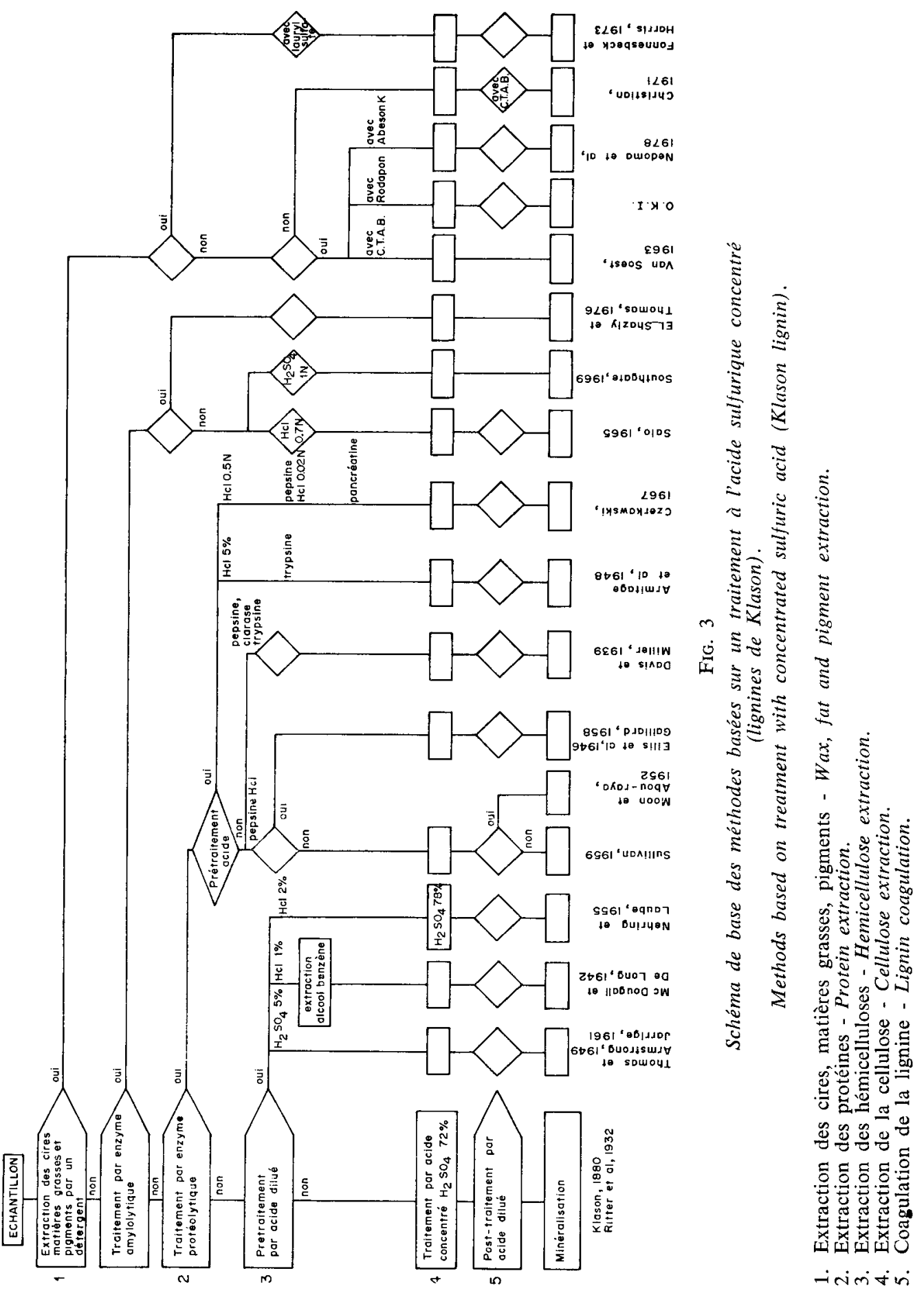


\& Maynard, 1946 ; Moon \& Abouraya, 1952 a ; Gaillard, 1958 ; Sullivan, 1959 ; FonNESBECK, 1976 (fig. 3). D'autres enzymes protéolytiques ont été employées : trypsine dans un milieu de carbonate de sodium (Armitage, Ashworth \& FERguSON, 1948) ou pancréatine (HALLSWORTH, 1950). CZERKAWSKr (1967) a fait agir de la pancréatine en plus de la pepsine, et Davis \& MiLler (1939) ont même employé trois enzymes pour le prétraitement : pepsine, clarase et trypsine. Les différentes enzymes utilisées proviennent d'organismes animaux dans lesquels elles interviennent à différents niveaux du tractus digestif.

Les travaux de Bevenue \& Williams (1959) ont mis en évidence l'aptitude de certains détergents à diminuer le taux azoté du résidu pariétal en plus de leur action sur les substances de nature lipidique. Ils sont à l'origine de différentes méthodes qui ne comprennent pas d'étapes enzymatiques dont les plus connues sont celles de Van Soest (1963), Fonnesbeck (1976), Nedoma, Stajgr \& Mika (1978) ou de l'Oskar Kellner Institute.

\section{c) Préhydrolyse}

La préhydrolyse par un acide minéral fort et dilué est une étape commune à presque toutes les méthodes puisque cette phase est seulement inexistante dans les méthodes anciennes de Klason (1880) et de MaHood \& Cable (1922) et dans celle proposée plus récemment par CHRISTIAN (1971). L'acide sulfurique est celui qui a été le plus employé à une concentration de 5 p. $100(\mathrm{P} / \mathrm{V})$ ou normale $(4,9$ p. 100 P/V) éventuellement en présence d'un détergent (Van Soest, 1963 et Nedoma, StajgR \& MIKA, 1978). Il a été remplacé par de l'acide chlorhydrique à des concentrations faibles : 1 p. 100 (McDougall \& De Long, 1942), 2,1 p. 100 (SPRINGER, 1949), 5 p. 100 (Armitage, Ashworth \& Ferguson, 1948), 0,5 N (Czerkawski, 1967) ou $0,7 \mathrm{~N}$ (SALO, 1965) (fig. 4).

extraction à chaud par l'éthanol

80 p. 100 à $90^{\circ} \mathrm{C}$

hot extraction with 80 p. 100 ethanol at $90^{\circ} \mathrm{C}$

$$
\downarrow
$$

empesage-autoclavage $1 \mathrm{~h}$ à $130^{\circ} \mathrm{C}$

attaque par glucoamylase à $\mathrm{pH} 4,7$ à $55^{\circ} \mathrm{C}$

incubation in autoclave for 1 hour at $130^{\circ} \mathrm{C}$

attack with glucoamylase (pH 4.7) at $55^{\circ} \mathrm{C}$

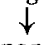

hydrolyse par $\mathrm{HCl} 0,7 \mathrm{~N}\left(5\right.$ heures à $\left.100^{\circ} \mathrm{C}\right)$

hydrolysis with $\mathrm{HCl} 0.7 \mathrm{~N}\left(5\right.$ hours at $\left.100^{\circ} \mathrm{C}\right)$

$\downarrow$

digestion avec $\mathrm{H}_{2} \mathrm{SO}_{4} 72$ p. $100\left(5\right.$ heures à $\left.20^{\circ} \mathrm{C}\right)$

digestion with 72 p. $100 \mathrm{H}_{2} \mathrm{SO}_{4}\left(5\right.$ hours at $20^{\circ} \mathrm{C}$ )

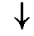

hydrolyse avec $\mathrm{H}_{2} \mathrm{SO}_{4} 1 \mathrm{~N}\left(4\right.$ heures à $100^{\circ} \mathrm{C}$ )

hydrolysis with $1 \mathrm{~N} \mathrm{H}_{9} \mathrm{SO}_{4}$ (4 hours at $100^{\circ} \mathrm{C}$ )

minéralisation

$\downarrow$

lignin

FIG. 4

Schéma de la méthode de Salo (1965).

Method of Salo (1965). 


\section{d) Hydrolyse principale}

Les méthodes comprennent généralement une hydrolyse à l'acide sulfurique très concentré $\left(\mathrm{H}_{2} \mathrm{SO}_{4} 72 \mathrm{p} .100 \mathrm{p} / \mathrm{p}\right.$ ou $\left.27 \mathrm{~N}\right)$ d'une durée variant entre deux et trois heures pour les méthodes concernant les fourrages ou les aliments concentrés (fig. 3 ). La lignine estimée gravimétriquement en tant que résidu organique de cette attaque est généralement appelée «lignine $\mathrm{H}_{2} \mathrm{SO}_{4} 72$ p. 100 » ou «lignine KLAson » du nom du chercheur qui, dès 1880 , l'a appliquée à des échantillons de bois.

SPRINGER a proposé en 1949 une méthode basée sur une hydrolyse avec de l'acide sulfurique à 78 p. 100 , c'est-à-dire à une concentration supérieure à celle généralement utilisée $\left(\mathrm{D}=1,704\right.$ à $\left.20^{\circ} \mathrm{C}\right)$. C'est cette méthode schématisée dans la figure 5 qui a été utilisée par NEHRING dans ses études sur les aliments concentrés (Nehring \& LAUBE, 1955).

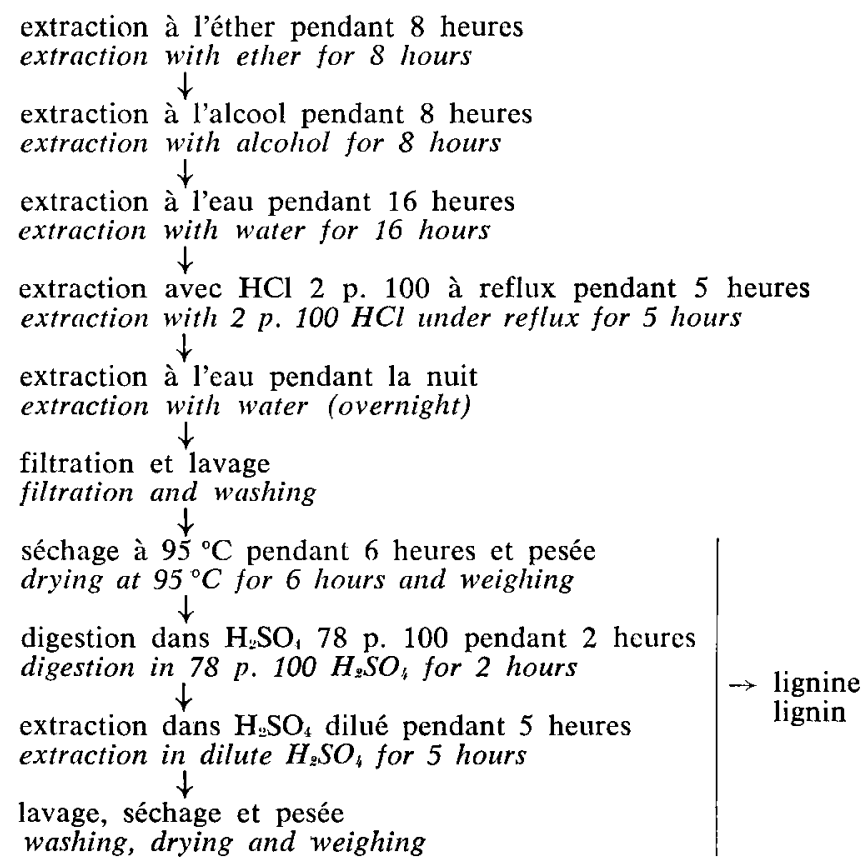

FIG. 5

Schéma de la méthode utilisée par Nehring (Nehring et Laube, 1955). Method used by Nehring (Nehring and Laube, 1955).

L'autre acide minéral fort et concentré qui a été utilisé est l'acide chlorhydrique. Ainsi, en 1913, Willstatter \& Zechtmerster ont proposé un traitement à l'acide chlorhydrique fumant $(\mathrm{d}=1,21)$ sur du bois. A cette concentration, l'acide chlorhy- 
drique dissout facilement la cellulose et les hémicelluloses et laisse la lignine sous forme d'un résidu insoluble. Cette méthode a été améliorée et utilisée par Krull (1916), Goss \& Phillips (1936) sur des échantillons moins ligneux (pailles, balles, épis) puis par Szentmilahyi (1972).

\section{e) Post-hydrolyse}

Dans la plupart des méthodes, le résidu obtenu après action de l'acide concentré est soumis à une post-hydrolyse avec de l'acide dilué par addition d'eau (concentration de 5 p. 100 (MaHood \& CABle, 1922, JARrige, 1961 a), figure 6, ou 3 p. 100 (RitTer, Seborg \& Mitchell, 1932). Cette dilution a été introduite en 1916 par Krull pour les méthodes basées sur une hydrolyse avec de l'acide chlorhydrique concentré.

\section{f) Conclusion sur les modes opératoires utilisés dans l'obtention des lignines KLASON}

Il existe donc de très nombreuses variantes de la méthode de KLASON utilisées en alimentation animale. Cependant, toutes les méthodes sont bâties sur le schéma décrit par Ellis, Matrone \& Maynard (1946), même si certaines étapes sont omises dans certains cas.

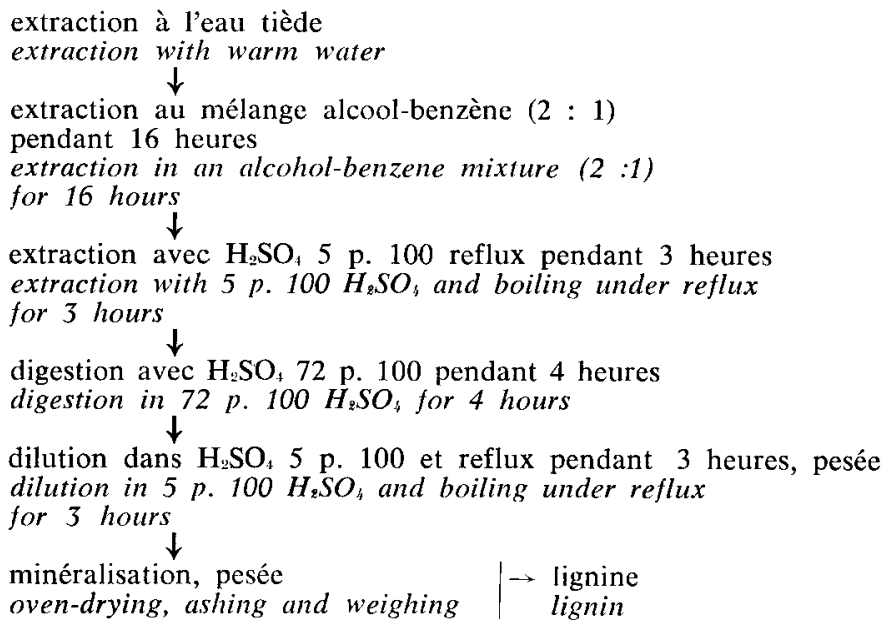

Deux méthodes utilisées en alimentation humaine pour estimer la lignine des produits céréaliers méritent d'être signalées : en effet, elles incluent dans le schéma précédent des phases de traitement par des enzymes amylolytiques : glucoamylase 
seule (Southgate, 1969) ou glucoamylase puis takadiastase (Elchazly \& Thomas, 1976). Ces traitements antérieurs à la préhydrolyse permettent, notamment, d'éviter la formation de produits de condensation entre la lignine et les glucides solubles.

\section{Méthodes basées sur l'extraction de la lignine}

Le principe de la mesure consiste à déterminer la lignine comme étant la perte de poids de l'échantillon au cours d'un traitement dit délignification.

\section{a) Par oxydation}

\section{- Par le chlore}

Les premières méthodes utilisées dans l'industrie papetière pour délignifier le bois résident dans la réaction du chlore sur la lignine et la formation de dérivés de substitution chlorés qui peuvent être éliminés par ébullition dans une solution de sulfite de soude (DoreE, 1949). C'est le principe de la méthode décrite par Cross \& Bevan (1903), reprise par Schorger (1917) qui a ajouté une phase de délipidation, puis par Goss \& Phillips (1936). Norman \& Jenkins (1933) ont utilisé l'action alternée et délignifiante du sulfite de sodium mélangé à de l'hypochlorite de sodium en milieu neutre puis acide sur des échantillons de bois.

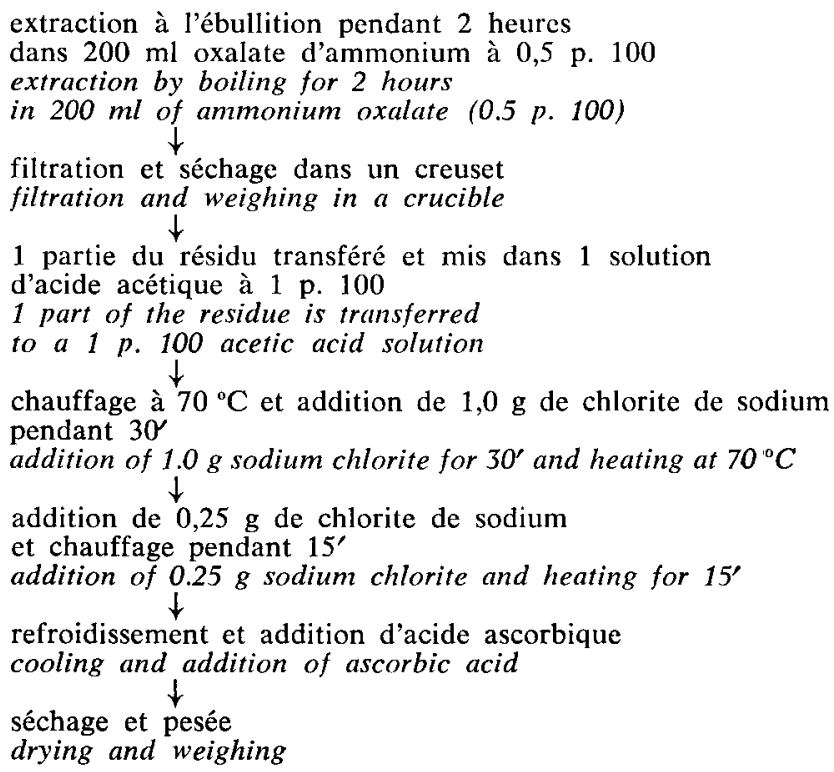

Lignine : fraction soluble dans le traitement au chlorite de sodium.

Lignin : fraction dissolved in sodium chlorite treatment.

FIG. 7

Schéma de la méthode de Collings et al. (1978). Method of Collings and al. (1978). 
Matrone, Ellis \& Maynard (1946) ont amélioré l'utilisation de cette méthode par l'addition d'une phase sodique. Dans toutes ces méthodes, les hémicelluloses sont éliminées par le traitement alcalin.

Enfin, Collings, Yokoyama \& Bergen (1978) utilisent une méthode au chlorite de sodium (fig. 7). Cette méthode a comme principe de base l'oxydation des cycles phényl de la lignine par le chlorite de sodium.

\section{- Par le permanganate}

Van SOEST \& Wine (1968) ont proposé une méthode dans laquelle, après un prétraitement de l'échantillon par une solution détergente en milieu acide (Van Solst, 1963) l'oxydation de la lignine se fait par action du permanganate de potassium (fig. 8). La « lignine permanganate » est estimée par la perte de poids liée au traitement par le permanganate sur le résidu issu du traitement par un détergent en milieu acide (ADF) (Van SoEST, 1963).

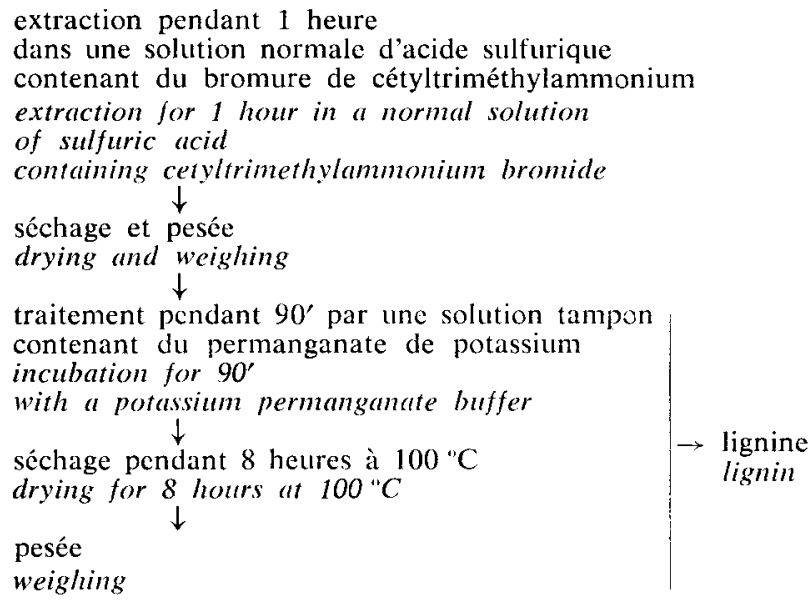

Fig. 8

Schéma de la méthode de Van Soest aut permanganate de potassium (Van Soest et Wine, 1968).

Method of Van Soest with potassium permanganate (Van Soest and Wine, 1968).

\section{b) Par disso'ution}

La méthode proposée par Edwards (1973) consiste en la dissolution de la lignine contenue dans le résidu ADF par un mélange de triéthylène glycol activé par de l'acide chlorhydrique (fig. 9).

\section{B. Méthodes non gravimétriques}

\section{Basées sur des propriétés optiques}

La lignine peut être solubilisće par un mélange de bromuic d'acétyle et d'acide acétique et présente dans ces conditions un spectre avec un pic d'absorption à 
$280 \mathrm{~nm}$. Cette propriété a été utilisée par Johnson, Moore \& ZanK (1961) et reprise par MORRISON (1972).

NorRIs et al. (1976) ont étudié la réflectance dans l'infrarouge d'échantillons de fourrages pour une gamme de longueurs d'ondes comprise entre 1,4 et 2,4 um. A partir de mesures faites parallèlement selon la méthode de Van Soest (Van SoEST, 1963 ; Van Soest \& Wine, 1968), Norris et al. (1976) ont établi des équations de prévision de cette lignine en fonction des résultats obtenus par réflectance.

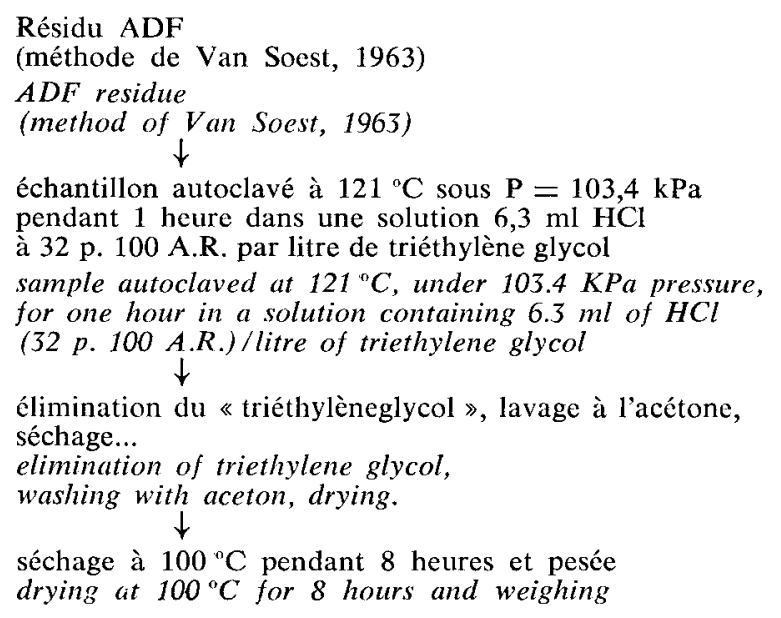

Lignine : perte de poids entre le résidu ADF et celui sortant de l'étuve.

Lignin : loss of weight between ADF residue and oven-dried residue.

FIG. 9

Schéma de la méthode d'Edwards (1973).

Method of Edwards (1973).

\section{Calorimétriques}

Schramm \& Bergner (1969) ont proposé d'estimer indirectement le contenu ligneux des aliments par une méthode calorimétrique.

Après extraction à l'eau et délipidation, un résidu dit lignocellulosique est obtenu par action de l'acide chlorhydrique $2 \mathrm{~N}$. L'échantillon est ensuite placé dans une bombe calorimétrique et sa chaleur de combustion mesurée. La teneur en lignine est estimée à partir de la valeur du résidu lignocellulosique déminéralisé et des chaleurs moyennes de combustion de la lignine $(6219 \mathrm{cal} / \mathrm{g})$ et de la cellulose (4123 cal/g) estimées auparavant. 


\section{Etude de la signification biochimique des différentes méthodes}

La plupart des méthodes utilisées en alimentation animale pour estimer la teneur en lignine des aliments sont de nature gravimétrique, done peu spécifiques. Il est donc important de connaître les principales limites de ces méthodes, et notamment de déterminer le degré de contamination des substances appelées « lignines " par d'autres comme les protéines ou les lipides ainsi que le degré de solubilisation des fractions constituant ces lignines, afin de juger de l'intérêt respectif des différentes méthodes et de leur utilisation. Un tel objectif est cependant difficile à atteindre car ces méthodes ont le plus souvent été proposées et utilisées par des zootechniciens dans un but d'application nutritionnelle, c'est-à-dire sans la nécessité d'obtenir des entités chimiquement pures. De plus, les méthodes ont été mises au point le plus souvent sur un faible nombre d'échantillons (moins d'une dizaine), généralement des fourrages, ce qui ne fournit pas une représentation significative des variabilités de composition pariétale des principaux types d'aliments distribués aux animaux domestiques. Cependant, à partir de certaines études méthodologiques plus approfondies, il est possible de cerner les principaux avantages ou défauts des méthodes les plus utilisées.

\section{A. Méthodes gravimétriques}

\section{Basées sur une hydrolyse en milieu acide concentré}

\section{a) Effets du séchage de l'échantillon}

Les échantillons, et plus particulièrement les produits humides comme les fourrages verts, sont séchés au moment de la prise d'essai afin de pouvoir les conserver jusqu'au moment de leur analyse. Cette pratique peut présenter certains inconvénients dans le cas des méthodes de "lignine KLASON » car un séchage initial peut entraîner la formation d'artefacts par réaction de condensation du type MaILlard notamment entre les glucides et les protéines (ADRIAN, 1974). Cet effet est d'autant plus marqué que la teneur en eau de l'échantillon avant séchage est élevée (Thomas \& Armstrong, 1949 et Moon \& Abou-Raya, $1952 \mathrm{~b}$; McDougall \& De Long, 1942 ; Van Soest, 1965), que la température est élevée (Thomas \& Armstrong, 1949 ; Van Soest, 1965 ; Streeter, 1969 ; Goering, Van Soest \& Hemken, 1973 ; Yu, 1976) et que la taille des particules est faible (Martilotti, 1973; Heller, Rivers \& Hackler, 1977). La nature de l'échantillon influe également, car l'effet de la température de séchage est d'autant plus marqué que l'échantillon est riche en matières azotées (cas des fourrages jeunes; Guessous, 1983) ou en substances pectiques (GIGER, données non publiées). Cette pratique de séchage avant analyse devrait donc être remplacée par la technique de lyophilisation, qui présente cependant l'inconvénient d'être coûteuse.

Ces artefacts de types «lignines» peuvent être estimés par la teneur résiduelle en azote du " résidu insoluble dans l'acide concentré » ou « lignine brute » (JARRIGE, 1961 a). Cette détermination de l'azote résiduel peut présenter un certain intérêt nutritionnel dans le cas où le blocage de l'azote est dû, non pas à un traitement de l'échantillon après la prise d'essai, mais à un traitement technologique qui peut 
modifier la valeur nutritionnelle d'un aliment (cas de la surchauffe pour les farines de luzerne déshydratées). Dans ce cas, la notion de résidu insoluble dans de l'acide concentré qui, d'un point de vue biochimique ne correspond pas à la lignine vraie, peut être d'un point de vue nutritionnel plus intéressante que celle de lignine vraie puisque ce résidu est essentiellement constitué de substances inutilisables par l'animal : de la lignine et de l'azote bloqué.

\section{o) Effet d'une extraction par des mélanges à base d'alcool}

Cette étape, dite de dégraissage, est d'autant plus nécessaire que les échantillons sont riches en tanins (RitTer, Seborg \& Mitchell, 1932 ; Shrikande, 1940) ou en extrait éthéré (Van SOEST, 1963).

Il semble que le mélange éthanol-benzène soit plus adapté à cette phase de dégraissage pour la préparation du résidu ligneux que l'éthanol seul, car le mélange éthanol-benzène dissoudrait en moins grande quantité les fractions dites ligneuses (Chastaignet, 1948 ; Moon \& Abou-Raya, 1952 a ; Jarrige, 1961 b). L'effet du dégraissage sur le dosage de la lignine est difficile à mettre en évidence puisque l'intensité du dégraissage peut influer sur la quantité de matière sèche extraite pendant cette phase, mais ne pas avoir d'influence significative sur le taux de lignine comme JARRIGE l'a mis en évidence sur des fourrages (JARRIGE, 1961 b).

L'utilisation de détergents dans la méthode de Van Soest (1963) permet d'éliminer cette phase d'extraction du moins dans le cas des fourrages. En effet, avec les aliments concentrés, le problème est à reconsidérer et l'échantillon doit subir une délipidation préalable lorsque le taux en extrait éthéré dépasse 5 p. 100 (Giger et al., données non publiées).

\section{c) Interférence avec les protéines}

Les travaux de Norman \& Jenkins (1934) ont mis en évidence la diminution de l'interférence entre les protéines et les glucides (en particulier les pentoses) due à l'action d'un prétraitement acide en milieu sulfurique normal. Ce prétraitement qui réduit la teneur en azote de la lignine brute entraînerait une perte en lignine selon différents auteurs (COHEN \& Harris, 1937 ; Harris \& Mitchell, 1939; Moon \& Abou-Raya, 1952 b; Kim, Gillingham \& LOAdholt, 1967).

Les traitements utilisant des enzymes protéolytiques ont permis de réduire le taux de protéines résiduelles de la lignine. Ils ne sont cependant pas totalement efficaces (Armitage, Ashworth \& Ferguson, 1948 ; Journet \& Jarrige, 1962), et, dans certains cas, ont conduit à des pertes en lignine notamment pour les préparations enzymatiques utilisées en milieu basique : pancréatine (CRAMPTON \& MAYNARD, 1938) ou trypsine (Journet \& JARRIge, 1962). La pepsine en milieu chlorhydrique $(0,1 \mathrm{~N})$ semble être l'enzyme la plus adaptée sans être totalement satisfaisante (JourNet \& JARRIGE, 1962).

L'utilisation de détergents (Van SoEST, 1963) permet de réduire le taux de protéines résiduelles, mais l'élimination des substances azotées n'est pas complète (MoIr, 1972).

Ces observations ont incité MorRison (1973) à combiner les deux procédés : utilisation d'un détergent et d'une préparation enzymatique. 
Une des variantes de la méthode de Van Soest (1963) d'obtention de la lignine consiste à faire deux hydrolyses successives à ébullition : la première avec une solution contenant un détergent (le laurylsulfate de sodium) en milieu neutre (Van SoEst \& Wine, 1967) et la seconde avec du bromure de cétyltriméthylammonium en milieu d'acide sulfurique normal (Van SoEsT, 1963) avant l'hydrolyse par l'acide sulfurique concentré ( 72 p. 100 p/v). Cette méthode a été critiquée car elle conduirait à des pertes importantes en lignine (RIOUET, 1979), qui peuvent s'expliquer par l'utilisation de la méthode initiale proposée par Van Soest \& WINE (1967) avec du sulfite de sodium employé pour son action déprotéinisante. Or, cette substance possède des propriétés délignifiantes (NoRMAN \& JENKIns, 1933) mises à profit en industrie papetière.

La comparaison des résultats obtenus avec six matières premières de nature très variée a montré qu'il n'y a pas de différence significative entre les valeurs de lignine obtenues avec la méthode par enchaînement (sans addition de sulfite de sodium lors de l'hydrolyse au détergent neutre) et celle avec uniquement une préhydrolyse avec le réactif au détergent acide (Acid Detergent Solution) (GIGER et al., données non publiées).

Comme il semble impossible d'obtenir une lignine exempte de toute contamination azotée du fait de la liaison étroite de fractions azotées à la lignine (BONDI \& MEYER, 1948 ; Preston, 1979), certains auteurs ont déterminé le taux azoté du résidu ligneux afin de le « corriger » (Thomas \& ARMSTrong, 1949 ; Journet \& JARrige, 1962).

Thomas \& Armstrong (1949) opèrent cette correction en multipliant le taux azoté estimé par la méthode KJELDAHL par le coefficient 6,25, ce qui implique que l'azote mesuré est inclus dans des molécules protidiques, ce qui n'est pas toujours le cas. Ainsi, les produits des réactions de MaILI.ARD qui sont des molécules dénaturées par la chaleur et qui peuvent contaminer la lignine ne contiennent en général pas 16 p. 100 d'azote. De ce fait, certains auteurs mettent en doute le bien-fondé de ce facteur de correction, et donc de son principe en lui-même (NoRMAN \& JENKINS, 1934 ; Moon \& Abou-Raya, 1952 b ; Salo, 1957).

\section{d) Influence de la préhydrolyse}

Cette phase opératoire permet d'éliminer un certain nombre de sucres qui contribuent à la constitution des hémicelluloses et qui peuvent former des produits de condensation avec la lignine au moment du traitement par l'acide sulfurique concentré (Norman \& JenKIns, 1934). Cependant, il semble qu'il soit très difficile et, voire même impossible, de définir les modalités d'une hydrolyse acide qui permette d'extraire la totalité des xylanes sans attaquer la cellulose tout au moins dans le cas où la lignine n'est pas extraite auparavant (JARRIGE, 1961 a).

Si on prend la méthode d'Ellis, Matrone \& Maynard (1946) comme référence, le traitement au "détergent acide " (Van SoEST, 1963) entraînerait une perte par dispersion colloïdale de 40 p. 100 environ de la lignine sur des échantillons de foin, paille et fécès. Par contre, ce traitement présente l'avantage d'éliminer les tannins qui peuvent contaminer les lignines dites de Klason (Porter \& Singleton, 1971).

Dans la méthode de SALo (1965), la préhydrolyse à l'acide chlorhydrique ne semble pas pouvoir permettre d'éliminer de manière satisfaisante les substances interférentes comme certaines protéines dénaturées par la chaleur lors de la fabrication 
industrielle de certains tourteaux de colza ou de tournesol (Borgida \& Tollier, 1976; Gullbot et Tollier, 1976). La teneur en lignine brute des échantillons obtenue par l'acide chlorhydrique 2 p. 100 est généralement plus faible que celle issue d'une hydrolyse par l'acide sulfurique 5 p. 100 (HARRis \& Mitchell, 1939).

\section{e) Traitement principal}

Les différents auteurs ne sont pas d'accord sur la durée de l'hydrolyse principale : il scmble qu'après une période minimale d'action de deux à trois heures à température ambiante ( 15 à $20^{\circ}$ C) (Norman \& Jenkins, 1934 ; Ritter, Seborg \& Mitchell, 1932), il y ait condensation ou caramélisation de certaines protéines ou de certains glucides dissous qui redeviendraient alors insolubles et augmenteraient artificiellement le taux « de résidu insoluble dans l'acide", (Ritter, Seborg \& Mitchell, 1932). En extrapolant les résultats issus de ces études sur les fourrages, une durée d'hydrolyse de deux à trois heures devrait suffire pour les aliments concentrés puisque ces échantillons contiennent en génćral peu de lignine.

La concentration de l'acide en elle-même peut jouer un rôle important sur la valeur du résidu obtenu (Fischer, 1961 a) : une concentration trop faible ne permet pas la solubilisation complète de la cellulose tandis qu'une concentration trop forte peut entraîner un processus d'humification (MoON \& ABOU-RAYA, 1952 a).

\section{f) Effet d'un post-traitement}

Les différentes études ne permettent pas de conclure définitivement sur l'utilité d'un post-traitement, ni sur ses modalités. Ainsi, Van SoEst (1963) l'a éliminé dans sa méthode. D'autres le considèrent comme indispensable (NORMAN \& JiNKINS, 1933 ; Ellis, Matrone \& Maynard, 1946 ; Moon \& Abou-Raya, 1952 a ; Salo, 1957 ; Porter \& Singleton, 1971 ; JARrige, 1980). Le post-traitement a pour effet de compléter l'hydrolyse et de dissoudre la lignine selon Ellis, MATronE \& MAYNARD (1946) et SALO (1957). Pour certains auteurs, il vaut mieux filtrer avant de diluer pour éviter une recondensation de la lignine avec les produits cellulosiques ou humiques qui peuvent précipiter (MOON \& Abou-Raya, 1952 b; Porter \& Singleton, 1971). Cependant si le dosage de la «lignine » doit représenter la quantité de substance indigestible et non celle de "substance insoluble dans les acides très concentrés", il vaut mieux ne pas filtrer (MoON \& ABOu-RAYA, 1952 b). D'après ces mêmes auteurs, il y a une perte en résidu dit ligneux si la dilution est effectuée sur un échantillon humide et non sur un échantillon préalablement séché.

\section{g) Correction par la teneur en cendres}

Le résidu obtenu par hydrolyse à l'acide sulfurique 72 p. 100 contient des matières minérales. D'après Moon \& ABou-RAYA (1952 a), les valeurs obtenues pour le résidu déminéralisé seraient beaucoup plus répétables que celles obtenues avec un résidu non déminéralisé. Il semble qu'une des raisons de cette différence de répétabilité soit due au fort pouvoir de rétention de l'acide sulfurique concentré par le résidu ligneux (POPOFF, 1938). La correction par la teneur en cendres permet donc de supprimer les éventuelles différences liées à l'intensité du lavage. De plus, cette correction est nécessaire pour les échantillons contaminés par de la terre dont les minéraux ne sont pas dissous dans les prétraitements (AERTs et al., 1978). 
h) Comparaison de quelques méthodes de lignine après hydrolyse en milieu acide concentré

\section{- Méthode de Jarrige (1961) et Van Soest (1963)}

Ces deux méthodes diffèrent essentiellement par la présence d'un détergent (le bromure de céthyltriméthylammonium) dans la méthode de Van SoEst (1963) ainsi que l'absence de post-hydrolyse dans cette méthode. La teneur en lignine brute diffère de deux points sur un ensemble de 14 échantillons de fourrages (tabl. 1 et JARRIGE, communication personnelle). Cette différence est probablement liée à une moins bonne extraction des matières azotées par la méthode de JARRIGE, mais peut-être aussi par une perte en constituants dits ligneux avec l'utilisation des détergents puisqu'il y a en moyenne 0,5 p. 100 MS d'écart entre les deux lignines corrigées par leur teneur résiduelle en azote.

\section{Tableau 1}

Comparaison de résidus insolubles dans l'acide sulfurique 72 p. 100. Comparison of acid insoluble residues.

(Jarrige, communication personnelle) (Jarrige, personal communication)

\begin{tabular}{|c|c|c|c|c|c|c|}
\hline \multirow{2}{*}{$\begin{array}{l}\text { Fourrage } \\
\text { Roughage }\end{array}$} & \multicolumn{2}{|c|}{$\begin{array}{l}\text { Lignine JARRIGE (1961) } \\
\text { Lignin JARRIGE (1961) }\end{array}$} & \multicolumn{2}{|c|}{$\begin{array}{c}\text { Lignine Van SoEst } \\
\text { (1963) } \\
\text { Lignin Van SoEst } \\
(1963)\end{array}$} & \multicolumn{2}{|c|}{$\begin{array}{l}\text { Matières azotées } \\
\text { (en \% lignine) } \\
\text { Crude protein } \\
\text { (\% lignin) }\end{array}$} \\
\hline & $\begin{array}{l}\text { brute } \\
\text { crude }\end{array}$ & $\begin{array}{l}\text { corrigée } \\
\text { corrected }\end{array}$ & $\begin{array}{l}\text { brute } \\
\text { crude }\end{array}$ & $\begin{array}{l}\text { corrigée } \\
\text { corrected }\end{array}$ & JARRIGE & VAN SOES'T \\
\hline Ray-grass . . & 4,01 & 2,36 & 2,93 & 2,52 & 41,2 & 11,6 \\
\hline Ray-grass ...... & 5,16 & 3,73 & 3,01 & 2,96 & 27,8 & 11,6 \\
\hline Dactyle ....... & 6,12 & 4,31 & 3,28 & 2,97 & 29,6 & 9,5 \\
\hline Dactyle .... & 6,40 & 3,85 & 3,31 & 2,82 & 39,7 & 14,7 \\
\hline Luzerne - Lucerne & 8,09 & 6,48 & 6,37 & 5,62 & 19,9 & 11,7 \\
\hline Luzerne - Lucerne & 8,20 & 6,30 & 6,82 & 6,14 & 23,9 & 11,4 \\
\hline Luzerne - Lucerne & 8,50 & 6,90 & 7,04 & 6,36 & 20,7 & 10,6 \\
\hline Luzerne - Lucerne & 9,00 & 7,24 & 8,29 & 8,01 & 19,5 & 7,5 \\
\hline Foin de luzerne ... & 9,27 & 7,70 & 7,72 & 7,16 & 17,0 & 7,3 \\
\hline Lucerne hay & & & & & & \\
\hline Foin de prairie & 7,50 & 6,28 & 4,73 & 4,20 & 12,6 & 9,9 \\
\hline Meadow hay & & & & & & \\
\hline $\begin{array}{l}\text { Foin de prairie } \\
\text { Meadow hay }\end{array}$ & 8,29 & 6,89 & 5,47 & 4,94 & 11,9 & 9,1 \\
\hline $\begin{array}{l}\text { Foin de prairie } \\
\text { Meadow hay }\end{array}$ & 7,98 & 6,46 & 6,63 & 6,01 & 19,1 & 9,4 \\
\hline $\begin{array}{l}\text { Foin de prairie } \\
\text { Meadow hay }\end{array}$ & 9,29 & 8,20 & 6,50 & 6,04 & 11,7 & 7,1 \\
\hline $\begin{array}{l}\text { Foin de prairie } \\
\text { Meadow hay }\end{array}$ & 10,25 & 9,09 & 8,25 & 7,65 & 11,3 & 7,2 \\
\hline$\overline{\mathbf{x}} \ldots \ldots \ldots$ & 7,72 & 6,13 & 5,74 & 5,24 & 21,8 & 9,9 \\
\hline$\sigma_{x} \ldots \ldots \ldots \ldots \ldots \ldots$ & 1,73 & 1,89 & 1,96 & 1,87 & 9,7 & 2,2 \\
\hline
\end{tabular}


- Méthodes de Van Soest (1963) et CHRISTIAN (1971)

Ces deux méthodes diffèrent uniquement par l'ordre de succession des hydrolyses acides : l'hydrolyse par l'acide sulfurique normal en présence de détergent acide (bromure de cétyltriméthylammonium) précède l'attaque par l'acide sulfurique concentré $(72 \mathrm{p} .100 \mathrm{p} / \mathrm{p})$ pour la méthode proposée par Van SoEst (1963), alors que la méthode de Christian (1971) commence par cette attaque à l'acide sulfurique 72 p. 100.

Ces deux méthodes diffèrent uniquement par l'ordre de succession des hydrolyses acides : acide sulfurique normal contenant le détergent (CTAB), et acide sulfurique concentré (72 p. $100 \mathrm{p} / \mathrm{p})$.

La méthode de Christian (1971) conduit à des valcurs plus élevées que celle de Van SoEst (1963) et ce, d'autant plus que l'échantillon est riche en lignine (fig. 10). Elle permet donc de mieux discriminer les échantillons que la méthode de Van ¿oest, mais la comélation entre les deux séries d'échantillons est très élevée $(0,980$ pour $n=24)$ (Rowett Research Institute, 1981).

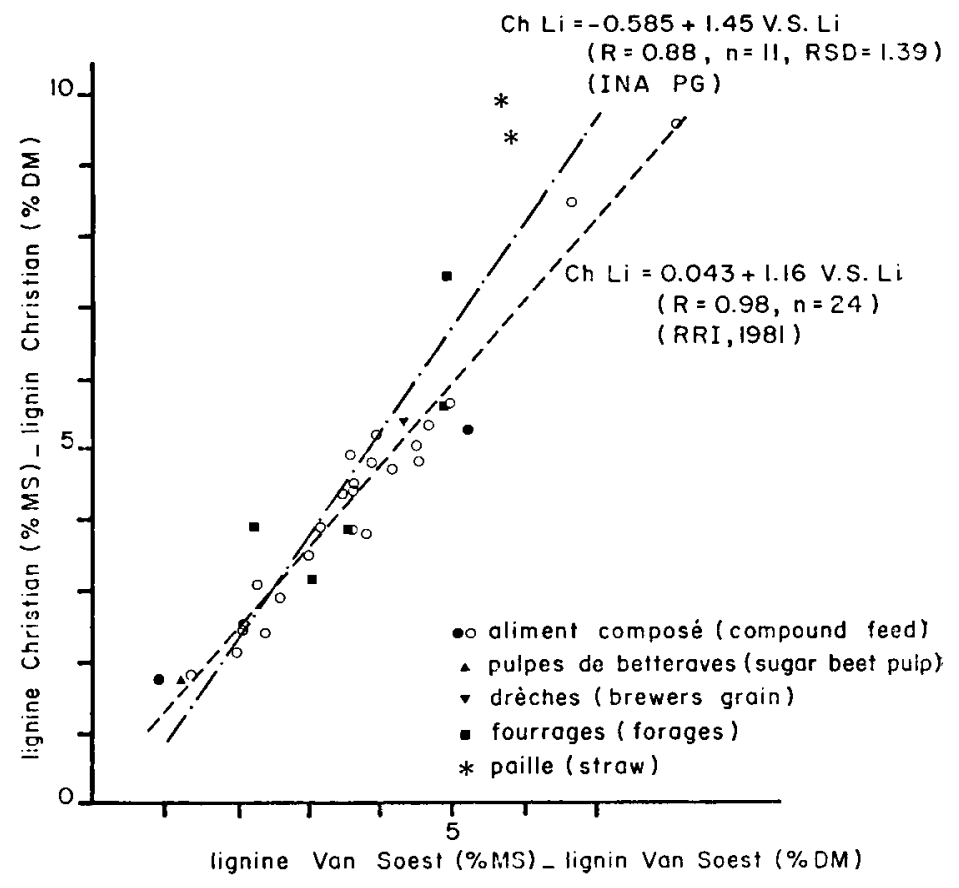

FIG. 10

Comparaison entre les teneurs en lignine

estimées selon les méthodes de Van Soest (V.S. Li) et de Christian (Ch. Li).

Comparison between lignin values estimated by the Van Soest (V.S. Li) and Christian (Ch. Li) methods. 
Cette différence entre les deux méthodes pourrait être liée à des pertes en lignine dans la méthode de Van SoEst (cf. étude précédente), soit à une élimination incomplète des produits de condensation formés à la suite de l'action directe de l'acide sulfurique concentré sur l'échantillon ainsi qu'une repolymérisation possible de lignine acidosoluble dans la méthode de Christian.

\section{- Méthodes de Van Soest (1963) et Nehring \& Laube (1955)}

Bien que la lignine, dans la méthode utilisée par (Nehring \& Laubi, 1955) soit obtenue avec de l'acide sulfurique concentré sur un échantillon ayant subi un prétraitement, les valeurs obtenues sont, pour des aliments de même nature et de même teneur en cellulose brute, plus élevées pour la méthode de NenRing \& Laube (1955) que pour celle de Van SoEst (1963). La différence est plus marquée pour les échantillons pauvres en fibre, c'est-à-dire riches en constituants cellulaires (fig. 11).

Les différences observées pourraient être dues à une moindre efficacité du traitement à l'acide chlorhydrique par rapport au bromure de cétyltriméthylammonium (CTAB) en milieu sulfurique normal ou à un début de processus d'humification avec de l'acide très concentré et des échantillons peu ligneux (MoON \& ABou-RAYA, 1952 b).

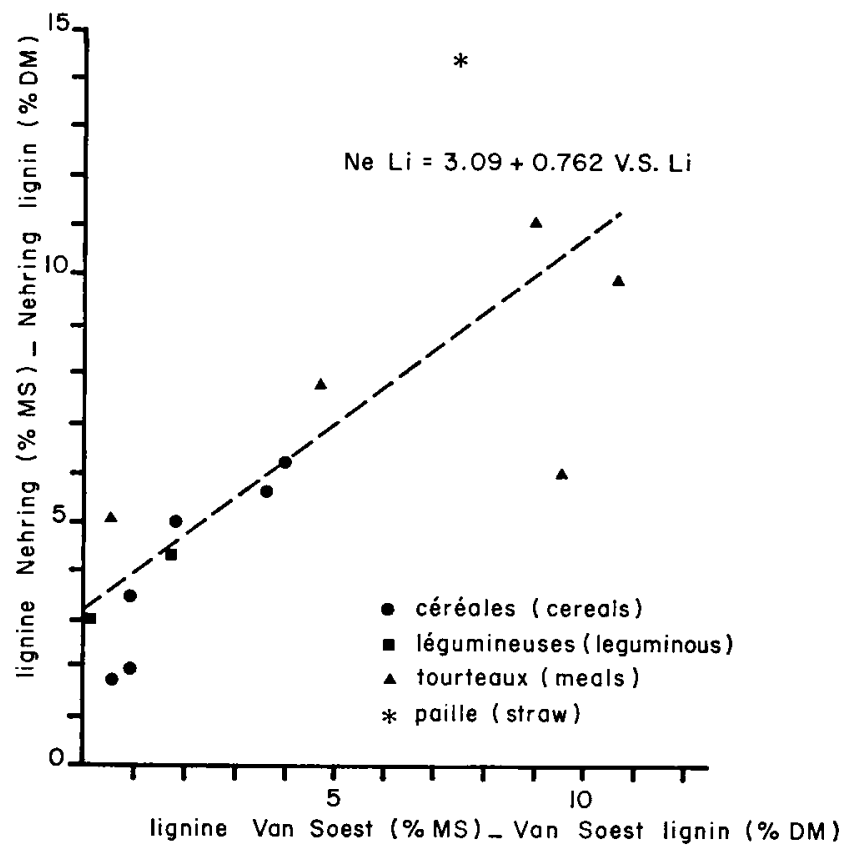

FIG. 11

Comparaison des valeurs obtenues pour les lignines de Van Soest (V.S. Li) et Nehring (Ne Li)

Comparison between values obtained with Van Soest (V.S. Li) and Nehring (Ne Li) lignins. 


\section{i) En milieu acide chlorhydrique concentré}

La lignine à l'acide chlorhydrique de Willstatter ne peut être débarrassée du chlore par lavage prolongé et est, de ce fait, surestimée de l'ordre de 1,5 à 2,0 p. 100 . Les valeurs obtenues par la méthode de Goss et PHiLlips (1936) seraient de plus supérieures à celles issues de la méthode à l'acide sulfurique du fait d'une repolymérisation pendant le traitement à l'acide chlorhydrique concentré sous forme acidoinsoluble d'une lignine acido-soluble (WHITEHEAD \& QurcKe, 1964).

Cependant, d'après Phillups \& Goss (1938) le résidu de « la lignine chlorhydrique » contiendrait moins de produits glucidiques condensés que la lignine « dite sulfurique ».

Bien que l'acide sulfurique concentré soit plus facile à conserver et à manipuler que l'acide chlorhydrique, cette dernière méthode a été jugée assez satisfaisante pour avoir été officialisée en 1955 par l'A.O.A.C.

\section{Méthodes basées sur l'élimination de la lignine}

\section{a) Par oxydation}

\section{- Par le permanganate}

La méthode d'oxydation de la lignine par le permanganate a été proposée par Van Soest \& Wine (1968) à partir du résidu ADF comme pour l'une des méthodes à l'acide sulfurique 72 p. 100 (Van SoEST, 1963). De ce fait, ces deux méthodes ont été comparées. Dans la méthode au permanganate, la "fraction lignine " comprend outre la lignine vraie, les tannins et exclut la cutine résistante à l'oxydation (Van SoEsT, 1969).

Par contre, dans la méthode à l'acide sulfurique concentré, le résidu ligneux comprend également de la cutine, des protéines dénaturées à la chaleur et des produits de condensation tannins-protéines. La lignine au permanganate reflète donc a priori mieux la teneur réelle en lignine de l'échantillon et correspond à une valeur inférieure à la lignine sulfurique sauf pour les échantillons riches en tannins (Van SoEST, 1969). La lignine au permanganate est une méthode un peu plus rapide que la précédente (Van SOEST \& Wine, 1968), mais plus délicate à mettre en œuvre (Giger, 1979), moins sensible aux traitements thermiques (Van SOEST \& WINE, 1968) mais plus sensible à la présence de polyphénols et autres substances insaturées comme les tannins, les pigments ou les protéines (Van SOEST \& WINE, 1968). Il y aurait perte par filtration d'un certain nombre de polysaccharides cellulosiques, notamment avec les échantillons immatures pauvres en lignine (Mertens, 1973 ; Southgate et al., 1976 ; Van Soest \& Robertson, 1979). Par contre, avec les échantillons riches en lignine, il peut y avoir manque de réactif et sous-estimation de la lignine (SouthGate et al., 1976). Sur un ensemble de 25 échantillons de nature très variée (fourrages, concentrés et fécès) (Van Soest, 1964, Giger et al., données non publiées), la corrélation entre les lignines sulfuriques et permanganate est à peine significative au seuil de 5 p. $100(\mathrm{r}=0,43$, $\mathrm{n}=25$ ). De même, Mertens (1973) montre que les coefficients de régression liant les lignines sulfuriques et permanganates sont satistiquement différents suivant les familles végétales considérées. 


\section{- Par le chlore}

Cette méthode est récente et a été encore peu étudiée. Les valeurs obtenues sont plus élevées que pour la lignine au permanganate (Collings, YokoYama \& BERGEN, 1978) : le permanganate serait un agent oxydant plus fort que le chlorite de sodium et pourrait attaquer les glucides pariétaux (lignine $\mathrm{KMn} 04=1,48+0,587$ lignine chlorite ; $\left.\mathrm{n}=0,54, \mathrm{n}=15 ; \mathrm{s}_{x y}=2,10\right)$.

La méthode au dioxyde de chlore semble difficilement applicable en dosage de routine du fait des risques que présente la manipulation de ce réactif.

\section{b) Par dissolution}

La méthode au triéthylène glycol donne des valeurs plus proches de la méthode au permanganate (Van SoEST \& WINE, 1968), que de la méthode à l'acide sulfurique concentré (Van Soest, 1963) du moins avec des échantillons de lupins, de trèfles et quelques graminées (EDwARDS, 1973). Sur ces mêmes échantillons (une vingtaine en tout), la précision des méthodes au permanganate et au triéthylène glycol est équivalente.

\section{B. Méthodes non gravimétriques}

\section{Basées sur des propriétés optiques}

La méthode au bromure d'acétyle de Morrison (1972) est une microméthode qui permet le dosage de la « lignine soluble dans les acides » (Tollier \& Riouet, 1980). Cependant, comme ces derniers auteurs l'indiquent, les trois points délicats du dosage sont l'élimination complète des polyphénols autres que les lignines, le choix d'une lignine de référence et la solubilisation totale de l'échantillon.

En effet, l'absorption à $280 \mathrm{~nm}$ qui constitue la base de cette méthode n'est pas liée à une entité chimique particulière. Il peut y avoir des interférences dues à des composées phénoliques ou des contaminations d'ordre humique (MORRISON, 1972).

La diminution de la ganulométrie de l'échantillon par broyage dans l'azote liquide ct une préextraction à température ambiante par de la soude normale sous azote pendant une heure constituent deux moyens qui permettent d'améliorer l'élimination des phénols et donc diminuer les sources d'interférences (Tollier, Riquet, 1980).

L'étalonnage de la gamme utilisée dans ce dosage se fait par référence à la teneur en lignine de KLASON ou à celle au dioxane. Cependant, il n'y a pas toujours accord entre les valeurs obtenues par la méthode au bromure d'acétyle étalonnée à partir de lignines au dioxane et celles issues de la méthode de KLAson (Tollier, RIQUET, 1980).

Le degré de solubilisation dans le bromure d'acétyle est un facteur important de la répétabilité qui est conditionnée pour une large part par la granulométrie des échantillons et la qualité ( « fraîcheur ») du réactif. Ce degré semble cependant variable suivant l'origine végétale (FAhey, McLaren,' Williams, 1979; Muntifering, 1982) et pour MCCONNELL \& EASTWOOD (1974), la méthode n'est pas applicable aux fruits et légumes. Cette méthode semble donc être difficilement applicable aux aliments composés utilisés en alimentation animale. 
La méthode de Norris et al. (1976) est une méthode rapide, mais sensible à la taille des particules et au séchage (SHENk, Westerhaus \& Hoover, 1979). Elle nécessite un étalonnage à partir d'au moins une cinquantaine d'échantillons. Comme il faut rechercher les longueurs d'onde appropriées à chaque type de fourrages ou d'aliments, cette méthode n'est pas bien adaptée au cas des aliments concentrés composés de formule souvent inconnue. De plus, ce procédé permet d'obtenir des résultats valables pour déterminer les teneurs en eau, matières azotées ou extrait éthéré, mais peu précis pour la lignine (WINCH \& MAJOR, 1981).

\section{Calorimétriques}

La méthode calorimétrique de Schramm \& Bergner (1969) peut être applicable à l'intérieur d'une même famille végétale, mais non à des aliments de nature aussi diverse que ceux entrant dans la fabrication des aliments concentrés, car la chaleur de combustion de la lignine est variable avec les différents types d'aliments (Schramm \& Bergner, 1969). Une explication de ce phénomène pourrait résider dans la variabilité de la structure de la lignine selon les aliments.

\section{Conclusion sur la signification biochimique des méthodes d'estimation de la lignine}

L'obtention du résidu ligneux par une méthode d'analyse chimique nécessite l'élimination préalable des substances susceptibles d'interférer. Il n'existe actuellement pas de méthode d'analyse qui permette d'obtenir une lignine pure quel que soit l'échantillon analysé et dont une fraction n'ait pas été dissoute. Une telle méthode semble d'ailleurs très difficile à concevoir puisqu'une simple hydrolyse à l'eau bouillante entraîne la rupture de liaisons intramoléculaires entre les lignines et les polyosides, donc la « perte » de complexes lignine-polyosides qui est évaluée à 5 p. 100 des pertes en constituants d'origine ligneuse avec des bois, c'est-à-dire des lignines considérées comme résistantes aux traitements (SARKANEN \& LuDWig, 1971). Toutes les méthodes d'estimation sont des compromis entre l'obtention d'une fraction pure et la destruction de composés ligneux. Il semble d'ailleurs difficile de concevoir une méthode qui soit parfaite pour tous les échantillons du fait de la variabilité de composition «des lignines ».

Cette étude a également montré que la méthode utilisée influait sur la valeur appelée «teneur en lignine » et que cette valeur ne correspond pas exactement à la teneur en lignine telle qu'elle est définie biochimiquement. Il nous semble donc préférable d'utiliser les termes de «lignine brute " ou de "résidu " suivis du nom de la méthode utilisée pour désigner les valeurs obtenues.

\section{Etude comparée de la signification nutritionnelle des principales méthodes de dosage de la lignine et de leur application pour l'alimentation des ruminants}

Lorsqu'il effectue un dosage de la « lignine », le nutritionniste cherche à estimer la part de l'indigestible dans la ration de l'animal, en particulier dans le cas du ruminant. De plus, il recherche une méthode qui soit applicable en grande série dans 


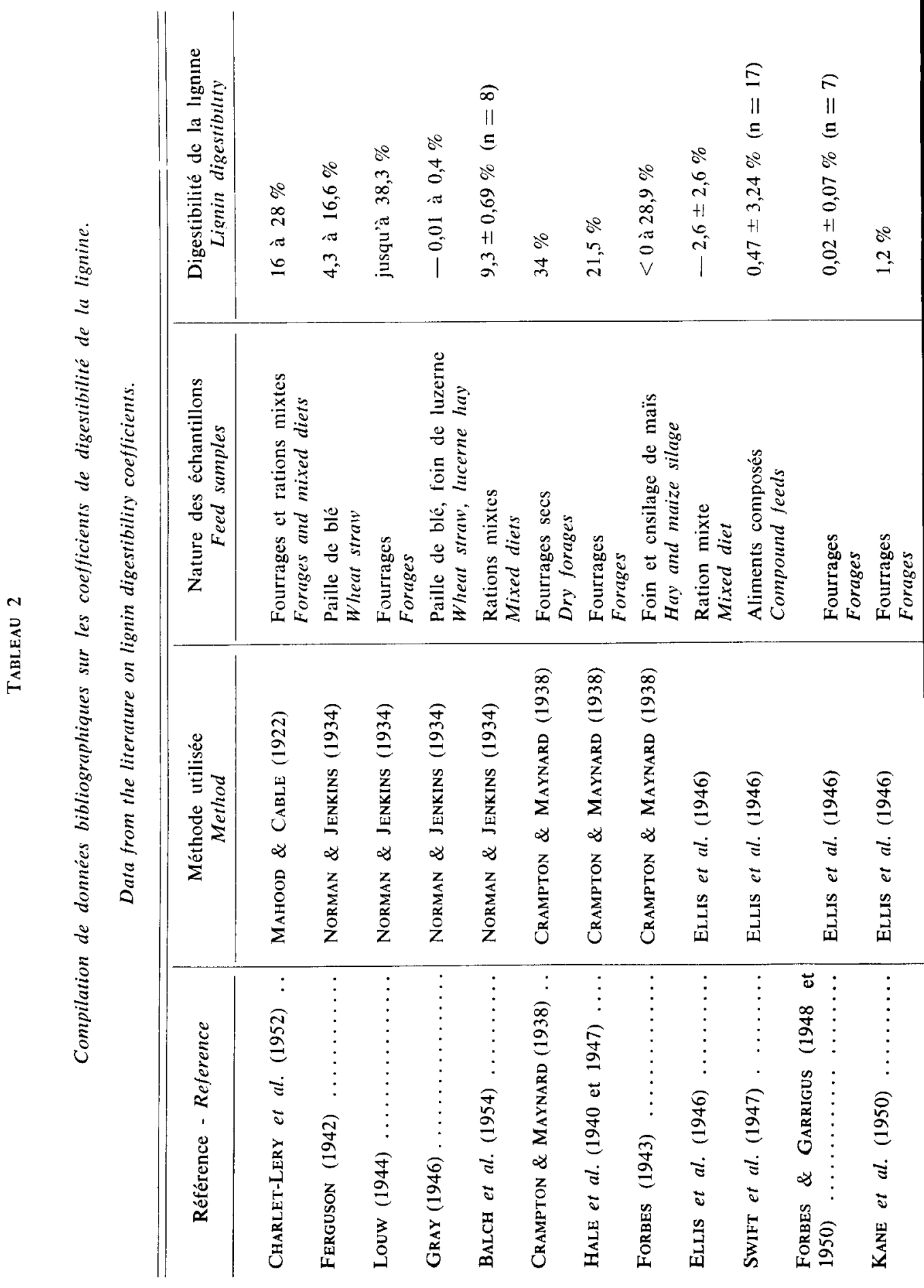


la plupart des laboratoires, c'est-à-dire répétable et reproductible, relativement simple et peu coûteuse et ne présentant pas de danger particulier.

\section{A. Etude comparée des valeurs de la digestibilité de la lignine obtenues avec différentes méthodes de dosage (tabl. 2)}

Si la lignine correspond à une fraction indigestible de la ration, sa digestibilité doit être nulle, c'est-à-dire que toute la fraction ingérée sous forme de lignine se retrouve au niveau fécal. Or, des phénomènes de déméthoxylation de la lignine ont été observés au niveau de l'estomac des monogastriques ou de la panse des ruminants par Csonka, Phillips \& Jones (1929) et François, Leroy \& Lery (1951). Hartley (1973) et GaILlard \& Richards (1975) ont montré que l'action des microorganismes du rumen pouvait entraîner la formation d'un complexe soluble lignine-glucide et contribuer ainsi à la dissolution de la moitié de la quantité totale de lignine ingérée, qui, de ce fait, n'est pas comptée sous forme de lignine au niveau fécal par les méthodes les plus couramment utilisées qui sont de nature gravimétriques, donc peu spécifiques en réalité (FaHEY, McLAREN \& Williams, 1979). En effet, il y a une différence importante d'un point de vue nutritionnel entre la dissolution et la digestion de la lignine, comme DekKer, Richards \& Playne (1972) l'ont souligné. MuntrFERING (1982) a émis deux autres hypothèses permettant d'expliquer des digestibilités positives de la lignine :

- les réactifs utilisés dans les méthodes de dosage de la lignine peuvent dissoudre partiellement la lignine fécale qui est un constituant «fragilisé » par son passage dans le tube digestif de l'animal;

- les aliments et les fécès peuvent se différencier des points de vue physique ou chimique dans la nature des constituants définis empiriquement par méthode gravimétrique sous le terme de "lignine » dans les schémas de méthode d'analyse des constituants pariétaux.

A l'opposé de ces différentes observations qui conduisent à des valeurs positives de la digestibilité de la lignine, il existe des raisons qui peuvent expliquer l'obtention des valeurs négatives de digestibilité de cette «lignine». Ces cas correspondent à la formation «d'artefacts de type lignine » dans le tube digestif de l'animal ou à une moindre élimination d'une substance qui, comme l'azote, peut interférer dans le dosage de la lignine suivant que ce sont les fécès ou l'ingéré qui sont dosés (LANCASTER, 1943 ; Forbes \& GARRIGUS, 1950).

Pour toutes les raisons qui viennent d'être développées et du fait des incertitudes expérimentales et méthodologiques, il est possible d'admettre que, d'un point de vue nutritionnel, les méthodes conduisant à des valeurs de «lignine » ou mieux de « résidu indigestible » comprises entre -10 et +15 p. 100 sont valables.

L'étude du tableau 2 montre que, pour une méthode donnée d'estimation de la lignine, les C.U.D. peuvent varier dans une large mesure suivant les auteurs : cas des méthodes de Norman \& Jenkins (1934), Crampton \& Maynard (1938), Springer (1949), Van Soest \& Wine (1968) et FonNesbeck (1976). En effet, il peut y avoir une interaction significative entre la fraction considérée comme lignine par une méthode donnée et le type d'échantillon considéré comme l'ont montré les études du Rowett Research Institute (R.R.I.) (1981) ou de Minson (1982).

Les méthodes les plus satisfaisantes d'un point de vue, nutritionnel sont, d'après la compilation de ces données, basées sur une hydrolyse en milieu d'acide sulfurique 
concentré (Ellis et al, 1946), Armitage et al. (1948), Journet \& IARrige (1962) et Van Soest (1963). Les deux premières ont même servi à une époque de calcul indirect des digestibilités de la matière organique selon la méthode des traceurs puisqu'elles donnaient des résultats de digestibilité de la lignine proches de zéro. La méthode proposée par Van SoEst (1963) présente l'avantage d'être plus rapide que les autres. ce qui explique son développement pour l'analyse des constituants pariétaux.

A notre connaissance, plusieurs des méthodes d'estimation de la lignine $n$ 'ont pas été utilisées lors d'expériences de digestibilité, ce qui limite les possibilités de conclure sur les intérêts respectifs des différentes méthodes d'estimation de la fraction "lignine " comme indigestible. Cependant, parmi les méthodes existantes non testées, celle de Morrison (1972) ne peut servir à des calculs de C.U.D. car la déméthoxylation de certains constituants de la lignine au cours des processus de la digestion modific les coclficients d'absorption des U.V. par les échantillons (ALLINSON \& OSBOURN, 1970). Baiba, Clarke \& Evans (1979) ont montré des pertes post-ruminales du caractère d'absorption des échantillons.

D’autre part, les valeurs de C.U.D. obtenues avec une méthode donnée peuvent varier dans une large mesure suivant la teneur en lignine de la ration. D'après les données obtenues avec la méthode de Van Soest (1963), il faut que la ration conticnne au moins 3 p. 100 de lignine pour que l'incertitude sur le C.U.D. ne soit pas trop importante (fig. 12, GIGER et al., données non publiées). Cette condition est généralement satisfaite dans les études citées précédemment car elles ont presque toutes porké uniquement sur des fourrages.

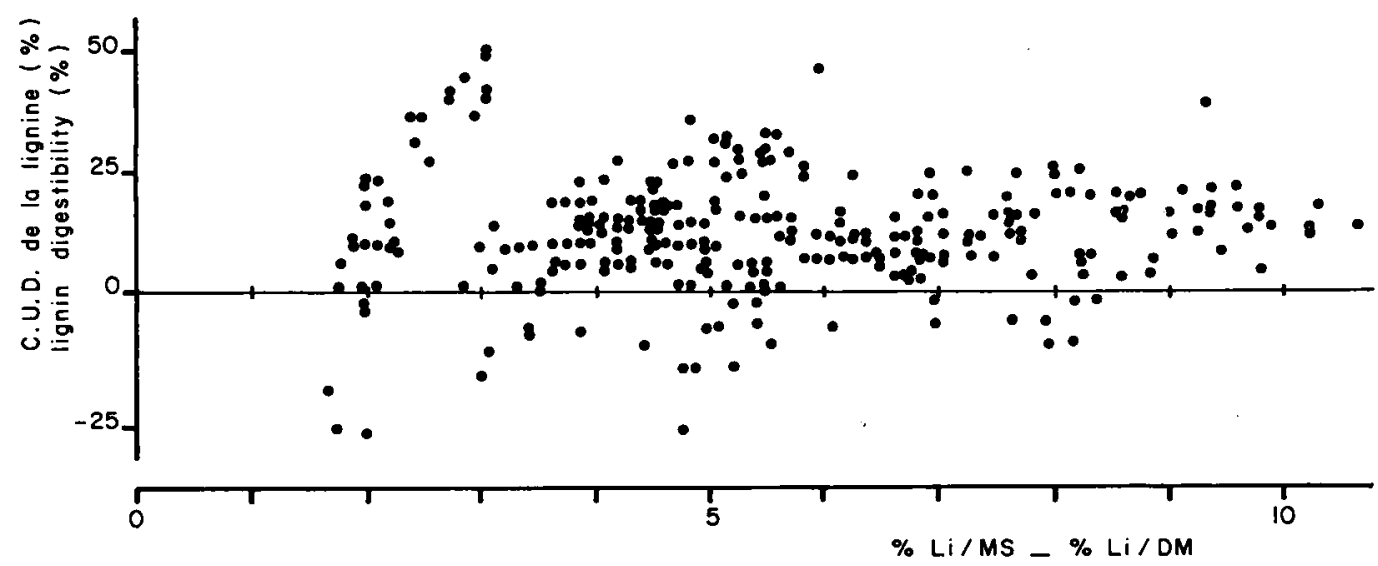

FIG. 12

Relation entre le coefficient d'utilisation digestive (C.U.D.) de la lignine et la teneur en lignine de la ration (Van Soest).

Relation between lignin digestibility coefficient and lignin content of the diet (Van Soest).

\section{B. Comparaison de différentes lignines}

sur la base du critère de la précision de la digestibilité des aliments

La comparaison des différentes méthodes de fractionnement de la paroi végétale en tant que prédicteur des facteurs de variation de la digestibilité des rations ou des 


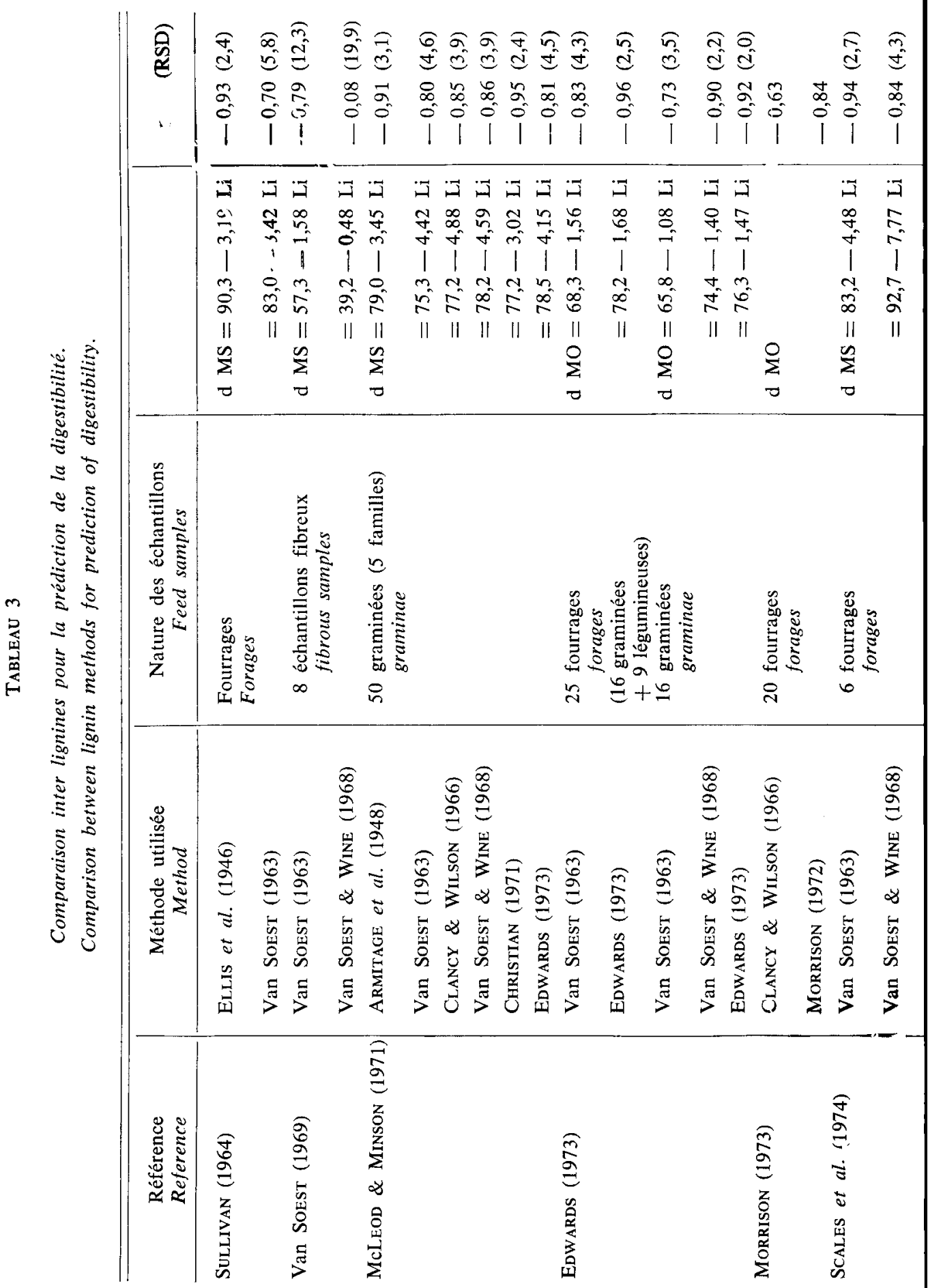


aliments pour les différentes espèces animales dépasse le cadre du présent travail. Cependant, la lignine considérée seule ou en association avec d'autres critères de paroi est un bon prédicteur de la variabilité de la digestibilité des aliments pour les ruminants. Du fait de la variabilité des résidus appelés lignine, il importe de chercher à comparer entre elles les différentes méthodes de dosage de la lignine utilisables pour prévoir les valeurs de digestibilité. Les comparaisons effectuées à notre connaissance ont été regroupées dans le tableau 2. Une étude analogue n'a pas été entreprise pour les monogastriques, car la digestibilité des aliments est essentiellement liée dans ce cas au taux en paroi végétale (Henry, 1971 ; King \& TAvernier, 1975).

Les comparaisons portent généralement sur les méthodes des lignines sulfurique et au permanganate de Van SoEST. Aucune hiérarchie nette ne se dessine entre les deux méthodes puisque les résultats de McLEod \& Minson (1971 et 1974), d'Edwards (1973), et d'AERTs et al. (1977 b) montrent que la lignine permanganate serait la meilleure, tandis que ceux de Van Soest (1969), de Scale et al. (1974) et de Mika et al. (1981) vont dans le sens inverse. Ces études ne portent pratiquement que sur des fourrages.

Les résultats sont également contradictoires lorsqu'on compare les lignines dites de Christian (1971) et de Van Soest (1963) (McLeod \& Minson, 1974 ; Rowett Research Institute, 1978 ; Giger et al., données non publiées). Il faut cependant noter que le coefficient relatif à la lignine au détergent acide de Van SoEst est plus grand que celui de la lignine de Christian, que ce soit pour la relation avec le coefficient d'utilisation digestive de la matière organique (tabl. 3) ou celui de l'énergie métabolisable des aliments concentrés en fonction de l'extrait éthéré (EE), des matières animales $(\mathrm{Mm})$ et d'une lignine (R.R.I., 1981) :

$$
\begin{aligned}
\mathrm{EM} / \mathrm{MS} & =13,07+0,249 \mathrm{EE}-0,109 \mathrm{Mm}-0,358 \mathrm{ch} \text { lignine }(\mathrm{RSD}=0,40 \mathrm{MJ}, \mathrm{n}=24) \\
& =13,16+0,244 \mathrm{EE}-0,123 \mathrm{Mm}-0,404 \mathrm{ADL} \quad(\mathrm{RSD}=0,43 \mathrm{MJ}, \mathrm{n}=24)
\end{aligned}
$$

Dans cette dernière étude, un accroissement de un point de la teneur en lignine réduit de 0,358 MJ et de 0,404 MJ la valeur en énergie métabolisable de l'aliment suivant la méthode utilisée : Christian ou Van Soest.

La méthode au triéthylène glycol d'Edwards (1973) serait meilleure dans une optique de prévision des coefficients d'utilisation digestive des rations que celles de Van Soest selon Edwards (1973) ou simplement équivalente (McLeod \& Minson, 1974). Il n'existe, à notre connaissance, qu'une comparaison sur 20 fourrages entre les méthodes de Morrison (1972) et de Van Soest (1963) modifiée par Clancy \& Wilson (1966). De ce fait, il est difficile de généraliser le résultat obtenu dans cette unique comparaison, c'est-à-dire la supériorité de la méthode aux U.V., d'autant plus qu'elle semble inapplicable, d'un point de vue pratique, aux aliments concentrés composés.

Sur une dizaine de matières premières (Giger, données non publiées) les précisions obtenues par les méthodes sulfuriques de Van Soest (1963) et NeHring \& NiePage (1955) semblent être proches. Cependant, récemment, NeHring (1979) a écrit que la méthode aux détergents devait supplanter la méthode qu'il utilisait. Ces comparaisons effectuées par approche statistique d'un ensemble de résultats cachent en fait des cas particuliers où une méthode est meilleure qu'une autre. Ainsi la teneur en lignine Christian (9,4 p. 100 de la MS) d'une paille de blé de teneur en cellulose brute de 43,1 p. 100 de la MS et de digestibilité in vitro de 44,8 p. 100 est de toute évidence plus cohérente que la teneur en lignine Van SoEST (1963) (5,9 p. 100 de la MS) (C.E.C., 1981). 
A l'issue de cette étude sur les potentiels comparés de prédiction du C.U.D. par la teneur en lignine, il n'est pas possible de préconiser une méthode car la hiérarchie entre les méthodes varie suivant les auteurs. Des études complémentaires de comparaison s'avèrent nécessaires sur ces aspects, d'autant plus que les échantillons concernés ici sont pratiquement tous des fourrages, et que certains ont été séchés à des températures élevées $\left(100^{\circ} \mathrm{C}\right)$ ce qui peut influer plus ou moins sur le taux de lignine selon la méthode utilisée.

\section{Faisabilité des méthodes}

Avant de procéder à des études comparatives plus poussées sur les méthodes de dosage de la lignine utilisables dans l'alimentation des ruminants, il importe de considérer la faisabilité des différentes méthodes. En effet, une méthode d'analyse doit, pour pouvoir être appliquée à grande échelle, être simple et peu coûteuse à mettre en œuvre, répétable et reproductible.

L'exigence de la simplicité de la méthode utilisée, bannit, parmi les méthodes chimiques, celles utilisées par Ellis, Matrone \& Maynard (1946), Nehring \& Niepage (1955) ou Journet \& Jarrige (1962). Par contre, l'introduction des détergents dans le schéma classique des lignines de KLASON a permis de bâtir des protocoles rapides de détermination de fractions dites ligneuses (Van SoEst, 1963; Van SoEst \& Wine, 1968 ; EDWARDS, 1973). L'obtention d'un résidu qui soit biochimiquement le plus pur possible conduit à retirer de la fraction résistante à l'hydrolyse par l'acide sulfurique concentré la part imputable à l'azote. Cependant, sur 26 échantillons de bersim, Guessous (1983) obtient des relations plus étroites entre le coefficient d'utilisation digestive de la matière organique et le taux de lignine, quand ce taux n'est pas corrigé par la teneur en MAT $(R=-0,938$ vs $-0,916$, et $\mathrm{Sxy}=1,71$ vs $1,98)$. Des points de vue nutritionnel et pratique, la correction du taux dinsoluble dans l'acide sulfurique 72 p. 100 par la teneur en MAT résiduelles ne semble donc pas à conseiller. Il faut néanmoins noter que ces résultats ont été obtenus sur des échantillons provenant d'une seule espèce végétale et séchés dans des conditions constantes $\left(70^{\circ} \mathrm{C}\right.$ pendant 48 heures). Dans d'autres conditions, une correction peut s'avérer nécessaire (séchage variable à température plus élevée).

Les méthodes basées sur des hydrolyses en milieu chloré concentré (Goss \& PhIllips, 1936) sont trop délicates à mettre en œuvre pour pouvoir être appliquées en routine à grande échelle (FISCHER, 1961 a). De plus, le chlorite de sodium utilisé dans la méthode de Collings \& Bergen, Yokoyama (1978) présente un danger d'explosion. La méthode au diméthylsulfoxide (TORP, 1980) est également à manipuler avec précaution. Les méthodes indirectes basées sur des mesures optiques qui sont séduisantes du fait de leur rapidité ne sont pas utilisables pour les aliments concentrés à cause du manque de réponse spécifique de la lignine aux rayons infra-rouges (NorRis et al., 1976) ou ultra-violets (MORRISON, 1973). Elles peuvent cependant présenter un certain intérêt pour les analyses de fourrages dont la structure de la lignine diffère peu au sein d'une même famille végétale. Pour des raisons analogues, la méthode calorimétrique de Schramm \& Bergner (1969) ne peut convenir du fait de la variabilité de la chaleur de combustion de la lignine.

La condition de bonne répétabilité des mesures effectuées conduit à préférer la lignine sulfurique à la lignine au permanganate (SCALEs et al., 1974; Giger, 1979). Ainsi, un écart-type résiduel de 0,27 points (p. 100 M.S.) a été obtenu entre deux 
séries de mesures effectuées sur un ensemble de 71 échantillons (GIGER et al., données non publiées). La méthode de la lignine sulfurique selon Van Soest est une méthode reproductible comme l'ont notamment montré Delort-Laval, BerTrand \& ValDEBOUzE (1980) et le compte rendu de l'enquête ITEB sur les aliments concentrés (SAUVANT et al., 1981) où l'écart-type résiduel entre deux laboratoires sur 64 échantillons est de 0,30 pour la lignine, c'est-à-dire équivalent à celui des cendres $(0,34)$ et nettement inférieur à celui sur la cellulose brute $(0,84)$. Ls résultats des chaînes d'analyse du B.I.P.E.A. (*) effectuées entre 1980 et 1982 montrent que l'écart moyen observé sur 25 échantillons analysés par une vingtaine de laboratoires différents est de 0,50 pour l'ADL.

Les méthodes d'estimation de la lignine dans les aliments destinés aux animaux, et plus particulièrement dans les aliments concentrés qui semblent être les plus faisables sont donc les méthodes d'analyse chimique. Parmi elles, il y a les méthodes dites de lignine de KLASON avec utilisation d'un détergent en prétraitement (Van SoEsT, 1963) ou post-traitement (CHRISTIAN, 1971) ou sans aucun détergent (MCDouGALL \& De LoNG, 1942).

Les méthodes au permanganate de Van SOEST \& WinE (1968) ou au triéthylène glycol d'EDWARDs (1973) méritent cependant d'être étudiées, même si leur mise en cuvre est un peu plus difficile que celle de Van Soest (1963).

\section{Conclusion}

La lignine étant une substance difficile à doser, il n'existe pas de méthode de référence qui permette de juger objectivement et de hiérarchiser les différents modes opératoires utilisés. Les valeurs obtenues peuvent varier d'une méthode à l'autre, ce qui implique de préciser systématiquement à côté du terme lignine la méthode ou la variante utilisée.

D'un point de vue biochimique strict, il vaudrait mieux utiliser des termes comme «lignines insolubles dans...» ou «fraction de lignine extraite par... » pour désigner la fraction considérée.

D'un point de vue analytique, aucune méthode n'est totalement satisfaisante dans la mesure où chacune est un compromis entre les pertes inhérentes aux différents traitements et la prise en compte des contaminations ou des formations d'artefacts. L'estimation du taux de lignine est considérée comme difficile pour le bois, mais le problème est encore plus complexe pour des substances qui en contiennent moins comme les fourrages et surtout pour la majeure partie des matières premières qui de plus sont riches en constitutants non pariétaux qui sont susceptibles d'interférer dans le dosage de la lignine.

D'un point de vue nutritionnel, aucune méthode d'estimation de la lignine ne semble être supérieure aux autres. Lorsqu'on considère que la lignine est un bon prédicteur de la digestibilité d'un aliment, le critère essentiel du choix d'une méthode de dosage de la lignine correspond donc actuellement principalement à la facilité de mise en œuvre du dosage au laboratoire, à sa répétabilité et à sa

${ }^{*}$ ) Bureau interprofessionnel d'Etudes analytiques, 145, Bourse de Commerce, Paris $\left(1^{\mathrm{ar}}\right)$. 
reproductibilité. Cette situation qui permet d'expliquer le succès actuel de la lignine au détergent acide de Van SoEST ne peut être jugée comme satisfaisante dans la mesure où les essais comparatifs n'ont pas été, à notre avis, suffisamment systématisés. Cette situation est d'ailleurs plus préoccupante dans le secteur des aliments concentrés et sous-produits pour lesquels les essais ont été les plus rares alors que l'existence. des mélanges commercialisés et de la grande variabilité des types de composition de paroi (Thivend, 1981 ; Sauvant, 1981) nécessitent plus que pour les fourrages. l'emploi d'une méthode de mesure du degré de lignification. Pour ces recherches, il conviendra de concevoir des dispositifs qui soient aptes d'une part à prendre en compte le maximum de "types» de lignine et, d'autre part, à dissocier efficacement l'influence du degré de lignification de celui de la teneur en paroi végétale. La satisfaction du premier objectif suppose de prendre en compte le maximum d'aliments, de familles, d'organes et de tissus végétaux différents, et celle du second à minimiser au départ la corrélation entre le degré de lignification de la paroi végétale: et la teneur en constituants pariétaux de nature glucidique.

\section{Remerciements}

Lauteur tient à remercier M. R. JARRIGE qui lui a fourni des résultats originaux et. MM. B. Monties et D. Sauvant pour leurs remarques lors de l'élaboration de ce texte.

\section{Summary \\ Review on lignin determination methods for feedstuff analysis}

Lignin is a non carbohydrate cellwall component whose structure is highly complex and variable according to feedstulfs. Because of this complexity and variability the term 《lignins » should be used rather than that of "lignin». Lignin is one of the main factors. determining the energy value of feedstuffs; being indigestible, it reduces the digestibility of the other cellwall components and is therefore important in the prediction of the dietary. energy content.

Most of the chemical methods are based on the scheme described by Ellis, Matrone. and MAYNARD (fig. l) using the ability of highly concentrated sulfuric acid to dissolve lignin. The main difference between the so-called KLASON lignins are the use of detergents (VAN: SoEst, 1963, fig. 2) or enzymes (amylases, proteases), replacement of sulfuric acid by hydrochloric acid (fig. 4), strength of the sulfuric acid concentration (fig. 3 and 5) or the. existence of a post-hydrolysis (fig. 3 and 6). In other methods, lignin is eliminated by means of chlore (fig. 7) or permanganate (fig. 8) or it is dissolved in a medium such as. triethyleneglycol. Indirect methods use optical properties (infrared reflectance or ultraviolet. absorption) or they are based on calorimetry.

However, a pure analytical fraction without any lignin loss or contamination by substances such as proteins, carbohydrates, cutins or tanins cannot be obtained with these methods (table 1). This methodological deficiency may be explained by the complexity and diversity of the socalled «lignin-fraction» which are generally highly correlated (fig. 10 and 11).

Comparisons between lignin estimation methods are scarce. Lignin digestibility is, seldom nul as it should be if this component was really indigestible (table 2, fig. 12). From data available until now, none of the methods seem to be better than others (table 3 ). Accordingly, further investigations should be made, especially with concentrates whose che-. mical composition is more variable than that of roughages.

Key words : lignin, review, estimation, feedstuffs, nutritive value. 


\section{Références bibliographiques}

AdRIAN J., 1974. Nutritional and physiological consequences of the Maillard reaction. World Rev. Nutr. Diet., 19, 71-122.

Aerts J.V., de Brabander D.L., Cottyn B.G., Buysse F.X., 1977 a. Comparison of laboratory methods for predicting the organic matter digestibility of forages. Anim. Fd Sci. Technol., 2, 337-350.

Aerts J.V., de Brabander D.L., Cottyn B.G., Buysse F.X., 1977 b. Contribution à la détermination de la valeur alimentaire des fourrages grossiers. IV. Estimation de la digestibilité et de la valcur énergétique à partir de la digestibilité réelle in vitro d'après VAN SoEst. Rev. Agric., 2, 437-453.

Aerts J.V., de Brabander D.L., Cottyn B.G., Buysse F.X., Carlier l.A., Moermans R.T., 1978. Some remarks on the analytical procedure of VAN SoEst for the prediction of forage digestibility. Anim. Fd. Sci. Technol., 3, 309-322.

AkIN D.E., 1979. Microscopic evaluation of forage digestion by ru:nen microorganisms. A review. J. Anim. Sci., 48, 701-710.

Allinson D.W., Osbourn D.F., 1970. The cellulose-lignin complex in forages and its relationship to forage nutritive value. J. Agric. Sci., 74, 23-36.

Armitage E.R. de B., Ashworth R., Ferguson W.S., 1948. Determination of lignin in plant material of high protein content. J. Soc. Chem. Ind. Lond., 67, 241-243.

Arroyo-Aguilu J.A., Evans J.L., 1975. Nutrient digestibility of forage and non forage rations in the ruminant. J. Agric. Univ. Puerto Rico, 51, 35-42.

Balba M.T., Clarke N.A., Evans W.C., 1979. The methanogenic fermentation of plant phenolics. Biochem. Soc. Trans., 7, 115-116.

Balch D.A., BaLCh C.C., Rowland S.J., 1954. Influence of the method of determination of lignin on the lignin-ratio technique for digestibility in the cow. J. Sci. Food Agric., 5, 584-588.

BeVenue A., Williams K.T., 1959. Note on the use of detergents for removal of nitrogen from plant materials. $J$. Assoc. Off. Anal. Chem., 42, 441-444.

Bondi A., Meyer H., 1948. Lignin in young plants. Biochem. J., 43, 248-256.

Borgida L.P., TOl.Lier M.Th., 1976. Le tourteau de colza exempt de thioglucosides ou détoxiqué par fermentation. I. Utilisation digestive des glucides et de l'énergie par le porc en croissance. Ann. Zootech., 25, 471-483.

C.E.C., 1981. C.E.C. workshop on methodology analysis of feedingstuffs for ruminants. European VAN SOEST ring test. Statistical report on analytical results. 34 p.

Charlet-lery G., François A., Leroy A.M., 1952. L'analyse des aliments destinés aux animaux et l'interprétation des résultats qu'elle fournit. Ann. Zootech., 3, 45-61.

Chastalgnet J., 1948. Study of extraction of wood with organic solvents. Mem. serv. Chim. Etat, 34, 255-262.

Christian K., 1971. Detergent method for total lignin in herbage. Fld. Stu. Rec. Div. Pl. Ind. CSIRO (Aust.), 10, 29-34.

ClanCY M.J., Wilson R.K., 1966. Development and application of a new chemical method for predicting the digestibility and intake of herbage samples. 10th Int. Grassland Congr., 445-453. Valtioneuvoston Kirjapaino ed., Helsinki, 1015 p.

Cohen W.E., Harris E.E., 1937. Pretreatment of wood with hot dilute acid. Effect on lignin values : Ind. Eng. Chem. Anal. Ed., 9, 234-235.

Collings G.F., Yokoyama M.T., Bergen W.G., 1978. Lignin as determined by oxydation with sodium chlorite and a comparison with permanganate lignin. $J$. Dairy sci., 61, 1156-1180.

Crampton E.W., Maynard L.A., 1938. The relation of cellulose and lignin content to the nutritive value of animal feeds. J. Nutr., 15, 383-395.

Cross C.F., Bevan E.J., 1903. Cellulose. London 102-104, cité par Hawley L.F. \& WISE L.E., 1931.

Csonka F.A., Phillips M., Jones D.B., 1929. Studies in lignin metabolism. J. Biol. Chem., 85, 65-75. 
CzerkaWsKı J.W.., 1967. The determination of lignin. Brit. J. Nutr., 21, 325-332.

Davis R.E., Miller C.O., 1939. Partition of the less easily digested carbohydrate complex of forages. Ind. Eng. Chem. Anal. Ed., 11, 651-652.

Di:kKer R.F.H., Richards G.N., Playne J.M., 1972. Digestion of polysaccharide constituents of tropical pasture herbage in the bovine rumen. - 1. Townsville stylo. Carbohydrate Res., 2E, 173-185.

Dhlor't-Laval J., Bertrand D., Valdebouze P., 1980. Test de reproductibilité des résultats du dosage de la cellulose WEENDE et des constituants membranaires selon VAN SOEST, B.I.P.E.A., janvier 1980, 108, 1-15.

Districh T., Konig J., 1871. Zusammensetzung und Verdaulichkeit der neben der Cellulose in der Rehfaser des Wiesenheus Vorkommenden Substanz. Landw. Vers. Sta., 13, 222-233, cité par FISCHER, 1961 b.

Dorer: Ch., 1949. Dosage de la collulose et de la lignine. In "Les méthodes de la chimie de la cellulose $\$ 376-399$. Dunod Ed., Paris.

EDWARDS C.S., 1973. Determination of lignin and cellulose in forages by extraction with triethylene glycol. J. Sci. Food. Agric., 24, 381-388.

Elchazly M., Thomas B., 1976. Über eine biochemische Methode zum Bestimmen der Ballaststoffe und ihrer Komponenten in pflanzlichen Lebensmitteln. Z. Lebensmittel Unters. Forsch., 162, 329-340.

Ellis G.H., Matrone G., MaYnaRd L.A., 1946. A 72 per cent sulfuric acid method for the determination of lignin and its use in animal nutrition. J. Anim. Sci., 5, 285-297.

Ely R.E., KANE E.A., JACOBSON W.C., MOORE L.A., 1953. Studies on the composition of lignin isolated from Orchard grass hay cut at four stages of maturity and from corresponding feces. J. Dairy Sci., 36, 346-355.

Fahey G.C. Jr, Mclarien G.A., Williams J.E., 1979. Lignin digestibility by lambs fed both low quality and high quality roughages. J. Anim. Sci., 43, 941-946.

Ferguson W.S., 1942. The digcstibility of wheat straw and wheat straw pulp. Biochen. $J$., 36, 786-789, cité par Fischtik, 1961 b.

Fischer H., 1961 a. Quantitative determination of lignin in hay. Acta Agric. Scand. Supplementuin Number 10, $43 \mathrm{p}$.

Fischer H.. 1961 b. Lignin and fecdstuff evaluation. A literature review. Kungl. Lantbrukshögsk. Ann., 27, 493-509.

FonNisbeck P.V., Harris L.F., 1973. A realistic system for partitioning food nutrients. J. Anina. Sci., $37: 279$ (abstr.).

FonNesfeck P.V., 1976. Estimating nutritive value from chemical analyses, 219-231 In P.V. Fonnesbeck, LE. Harris and L.C. Kearl (Ed.). First International Symposium, Feed Composition, Animal Nutrient Requirements and Computerization of diets. Utah Agr. Exp. Sta. Utah State Univ. Logan.

Fonnesbeck P.V., Christiansen M.L., Harris L.E., 1981. Linear models for calculating digestible energy for sheep diets. J. Anim. Sci., 52, 1183-1196.

Forbes E.B., 1943. Conditions affecting the digestibility and metabolizable energy of feeds for cattle. Pennsylvania Agr. Exp. Sta. Bull., 452, 34 p.

Fcrbes R.M., Garrigus W.P., 1948. Application of the lignin ration technique to the determination of the nutrient intake of grazing animals. J. Anim. Sci., 7, 373-382.

Forbis R.M., Garricus W.P., 1950. Some effects of forage composition on its nutritive value when cut and fed green to steers and wethers as determined conventionally and by the lignin ratio. J. Anim. Sci., 9, 531-539.

Françors A., Lleoy A.M., Lery G., 1951. Le sort des radicaux methoxy au cours de la digestion des pectines et des lignines par les bovins. C.R. Acad. Sci., 232, 1323-1325.

Fremy, 1883. Chimie biologique et chimie physiologique. In «Encyclopédie chimique», t. X p. 21, Dunod éd. Cité par Monties (1980).

FreUdenBERG K., 1965. Lignine : its constitution and formation from p-hydroxycinnamyl alcohols. Sciences, 148, 595-600.

GaIllard B.D.E., 1958. A detailed summative analysis of the crude fibre and nitrogen free extractives fractions of roughages. 1. Proposed schema of analysis. J. Sci. Food. Agric., 9, 170-177. 
Gaillard B.D.E., Richards G.N., 1975. Presence of soluble lignin-carbohydrate complexes in the bovine rumen. Carbohydrate Res., 42, 135-145.

Galyean M.L., Wagner D.G., Owens F.N., 1979 a. Level of feed intake and site and extent of digestion of high concentrate diets by steers. J. Anim. Sci., 49, 199-203.

Galyean M.L., Wagner D.G., Owens F.N., 1979 b. Corn particle size and site and extent of digestion by steers. J. Anim. Sci., 49, 204-210.

Grger S., 1979. Some aspects of assessments of plant cell wall constituents by the Van SoEST method. FOCUS, n ${ }^{\circ} 5,6-7$.

Goering H.K., Van Soest P.J., Hemken R.W., 1973. - Relative susceptibility of forages to heat damage as effected by moisture, temperature and pH. J. Dairy Sci., 56, 137-143.

Goss M.J., Pinllips M., 1936. Studies on the quantitative estimation of lignin. Factors affecting the determination by the fuming $\mathrm{Hcl}$ method. J. Ass. Off. Agr. Chem., 19, 341-350.

GraY F.V., 1946. The digestion of cellulose by sheep. The extent of cellulose digestion at successive levels of the alimentary tract. J. Exp. Biol., 24, 15-19.

Guessous F., 1983. Composition chimique et valeur nutritive du bersim (Trifolium alexandrinum L.). Thèse de doctorat d'état, ès-Sciences Naturelles, 22 mars 1983, Paris, 304 p.

Guilbot A., Tollier M.Th., 1976. Extraction et dosage de différents glucides de graines oléagineuses et de leurs dérivés. Etudes et recherches. 2. Revue Française des corps gras. $23^{\circ}$ année, 6, 323-330.

Hale E.B., Duncan C.W., Huffman C.F., 1940. Rumen digestion in the bovine with some observations on the digestibility od alfalfa hay. J. Dairy Sci., 23, 953-967.

Hale E.B., Duncan C.W., Hufrman C.F., 1947. Rumen digestion studies. J. Nutr., 34, $733-758$.

Hallsworth E.G., 1950. The crude fibre determination and its alternatives. Agr. Progr., $25,39-49$.

Harbers L.H., Brazle F.K., Raiten D.J., Owensby C.E., 1981. Microbial degradation of smooth brome and tall fescue observed by scanning electron microscopy. J. Anim. Sci., 51, 439-446.

HARRIS F., Mitchell H.H., 1939. Effect of various preextractions on the lignin determination of wood. Ind. Eng. Chem. Anal. Ed., 11, 153-155.

HARTLEY R.D., 1973. Carbohydrate esters of ferulic acid as components of cell walls of Lolium multiflorum. Phytochem., 12, 661-665.

Hawley L.F., Wise L.E., 1931. Détermination de la lignine, 174-185, in « La chimie du bois » traduit par Barry, J. Dunod éd. Paris, 362 p.

Heller S.N., Rivers J.M., Hackler L.R., 1977. Dietary fiber : the effect of particle size and $\mathrm{pH}$ on its measurement. J. Food. Sci., 42, 436-439.

Henneberg W., Stohmann F., 1860. Beiträge zur Begründung einer rationnellen Fütterung der Wicderkauer. Braunschweig, 1860, cité par FISCHER, 1961 b.

Henry Y., 1971. Essai de prévision de la valeur en énergie digestible des aliments pour le porc, à partir de leur teneurs en constituants membranaires. J. rech. porcine en France, I.N.R.A., I.T.P. éd. Paris, 57-64.

JARRIGE R., 1961 a. Analyse des constituants glucidiques des plantes fourragères. I. Fractionnement des constituants de la membrane par les hydrolyses acides. Ann. Biol. Anim. Biochim. Biophys., 1, 163-212.

JARRIGE R., 1961 b. Analyse des constituants glucidiques des plantes fourragères. II. La lignocellulose : composition, dosage et comparaison avec la cellulose brute. Ann. Biol. Anim. Biochim. Biophys., 1, 421-427.

JARRIGE R., 1980. Chemical methods for predicting the energy and protein value of forages. Ann. Zootech, $\mathrm{n}^{\circ}$ hors série, 299-324.

JARRIGE R., Minson D.J., 1964. Digestibilité des constituants du ray grass anglais S 24 et du dactyle S 37. Ann. Zootech., 13, 117-150.

Jentsch W., Wittenburg H., Schiemann R., Hoffmann L., 1969. Ergebnisse vergleichender Untersuchungen über die Verdaulichkeit von Rationen bei Rind und Schaf unter besonderer Berücksichtigung pansenphysiologischer Parameter. 2 - Mittelung : Untersuchungen 
von 12 Rationen mit stufenweisem Austausch von Rohfaser gegen $\mathbf{N}$ freie Extraktstoffe bsw. N freien Extrakstoffen gegen Rohprotein. Arch. Tierernähr., 19, 357-370.

Johnson D.B., MoOre W.E., ZANK L.C., 1961. The spectrophotometric determination of lignin in small wood samples. TAPPI Technical Section, 44, 793-798.

Journet M., JARrige R., 1962. Analyse des constituants glucidiques des plantes fourragères. III. Etudes sur le dosage de la lignine. Ann. Biol. Anim. Biochim. Biophys., 2, 223-250.

Kane E.A., Jacobson W.C., Moore L.A., 1950. A comparison of techniques used in digestibility studies with dairy cattle. J. Nutr., 41, 583-596.

Kim J.T., Gillingham J.T., Loadholt C.B., 1967. Difference in composition between crude fiber and acid-detergent fiber. J. Assoc. Off. Anal. Chem., 50, 340-343.

KIng R.H., TAVERNER M.R., 1975. Prediction of the digestible energy in pig diets from analyses of fibre contents. Anim. Prod., 21, 275-284.

KLASON, 1880. Bericht der Vereine der Papier und Zellstoffchemiker (1880), 52-53. Cité par HaWley et Wise (1931).

Krull, 1916. Versuche iiber Verzuckerung der Zellulose. Thèse, Dantzig. Cité par HawleY et WISE, 1931.

Lancaster R.J., 1943. Metabolism trials with New Zealand feeding stuffs. New Zealand J. Sci. Tech., 25, 137-141.

Louw J.G., 1944. The relative digestibility of the constituents of the carbohydrate complex of grasses at successive stages of growth with reference to their partition into crude fiber and nitrogen free extract according to the standard method for feedingstuff analysis. Onderstepoort J. Vet. Sci., 17, 165-179.

McConnell A.A., Eastwood M.A., 1974. A comparison of methods of measuring «fibre » in vegetal material. J. Sci. Food Agric., 25, 1451-1464.

McDougall D., De Long W.A., 1942. Effect of initial drying temperature on the apparent lignin content of plant tissues. Can. J. Res., $20 \mathrm{~B}, 40-48$.

McDougall D., De Long W.A., 1948. A method for determination of the lignin content of fresh plant tissue without preliminary drying. Can. J. Res., 26 B, 468-471.

MCLeod M.N., Minson D.J., 1971. The error in predicting pasture dry-matter digestibility from four different methods of analysis for lignin J. Br. Grassl. Soc., 26, 251-256.

MCLeod M.N., Minson D.J., 1974. The accuracy of predicting dry matter digestibility of grasses from lignin analyses by three different methods. J. Sci. Food Agric., 25, 907-911.

Mahood S.A., Cable D.E., 1922. The chemistry of wood. J. Ind. Eng. Chem., 14, 933-934.

Martilottri F., 1973. Studies on lignin. 1. Effect of fineness of grinding and temperature of drying of the sample on analytical results. Ann. Ist. sper. Zootec. Roma, 6, 151-166.

Matrone G., Ellis G.H., Maynard L.A., 1946. A modified Norman-Jenkins method for the determination of cellulose and its use in the evaluation of feedstuff. J. Anim. Sci., 5 , 306-312.

MerTens D.R., 1973. Application of chemical mathematical models to cell wall digestion and forage intake in ruminants. Ph. D. Thesis Cornell University, $217 \mathrm{p}$.

Mika V., Paul C., Zimmer E., Kaufmann K., 1981. Ein Vergleich verschiedener Labormethoden zur Schätzung der Verdaulichkeit von Grundfutter. Z. Tierphysiol. Tierernähr. Futtermittelkd, 45, 132-141.

Minson D.J., 1982. Effect of chemical composition on feed digestibility and metabolizable energy. Nutr. Abstr. Rev., 52 B, 591-615.

MoIR K.W., 1972. The effect of different extraction procedures on the recovery of cell walls in forage and faeces from cattle and sheep. J. Agric. Sci. Camb., 78, 351-353.

Monties B., 1980. Les lignines. In «Les polymères végétaux», 122-155. Ed. Gauthier Villars, Paris.

Monties B., Lapierre Catherine, 1981. Données récentes sur l'hétérogénéité de la lignine. Physiol. Vég., 19, 327-348.

Moon F., ABou-RaYA A.K., 1952 a. The lignin fraction of animal feeding-stuff. II. Investigation of lignin determination procedures and development of a method for \& Acidinsoluble lignin ». J. Sci. Food Agric., 3, 407-418. 
Moon F.E., AbOU-Raya A.K., 1952 b. The lignin fraction of animal feeding-stuff. III. The determination of «total lignin». J. Sci. Food Agric., 3, 595-608.

MORRISON I.M., 1972. A semi-micro method for the determination of lignin and its use in predicting the digestibility of forage crops. J. Sci. Food Agric., 23, 453-455.

Morrison I.M., 1973. The isolation of plant cell wall preparations with low nitrogen contents. J. Agric. Sci., 80, 407-410.

MORRISON I.M., 1979. Symposium on carbohydrate metabolism in the ruminant : carbohydrate chemistry and rumen digestion. Proc. Nutr. Soc., 38, 269-274.

MUNTIFering R.B., 1982. Evaluation of various lignin assays for determining ruminal digestion of roughages by lambs. J. Anim. Sci., 55, 432-438.

Nedoma J., Stajgr V., Mika V., 1978. Forage quality. 1. Study of detergents suitable for determining of crude fibre. Scient. Agric. Bohem., 10, 267-277.

Nehring K., Laube W., 1955. Untersuchungen über die Zusammensetzung der pflanzlichen Gerüstsubstanzen in Gruen und Rauhfutterstoffen und ihren Einfluss auf die Verdaulichkeit dieser Futterstoffe. Arch. Ticrernähr., 5, 177-215.

Nehring K., Niepage N., 1955. Die Bestimmung des Lignins in Futterstolfen. $Z$. Landwirtschaftl. Versuchs Untersuchungswesen. 1, 312-327.

Nehring K., 1979. Die Ermittlung des Futterwertes. Arch. Tierernähr, 29, 311-337.

Norman A.G., Jenkins S.H., 1933. A new method for the determination of cellulose, based upon observations on the removal of lignin and other encrusting materials. Biochem. J., 27, 818-831.

Norman A.G., JEnkins S.H., 1934. The determination of lignin. 2. Errors introduced by the presence of protein. Biochem. J., 28, 2160-2168.

Norris K.H., Barnes R.F., Moore J.E., Shenk J.S., 1976. Predicting forage quality by infrared reflectance spectroscopy. J. Anim. Sci., 43, 889-897.

Phillips M., Goss M.J., 1938. Effect of various carbohydrate materials on the determination of lignin by the fuming hydrochloric acid method. J. Ass. Off. Chem., 21, 140-145.

PopoFF I.D., 1938. Zur Methodik der quantitativen Ligninbestimmung. Z. Tierernähr Futtermittelk, 1, 245-259.

Porter P., Singleton A.G., 1971. The degradation of lignin and quantitative aspects of ruminant digestion. Br. J. Nutr., 25, 3-14.

Preston R.D., 1979. Polysaccharide conformation and cell wall function. Annu. Rev. Plant. Physiol., 30, 55-78.

RanFFT K., Kirchgessner M., Roth F.X., 1976. Detergentieanalysen zur Bestimmung von Gehalt und Verdaulichkeit der Zellwandfraktionen in Weidegras. Landwirtsch. Forsch., 29, $124-130$.

RiQUET A.M., 1979. Etude des constituants membranaires de divers produits végétaux destinés à l'alimentation animale. Signification et limites des principales méthodes de dosage. Thèse de $3^{\circ}$ Cycle, E.N.S.I.A., 29 octobre 1979, Paris, 93 p.

Ritter G.J., Seborg R.M., Mitchell R.L., 1932. Factors affecting quantitative determination of lignin by 72 per cent sulphuric acid method. Ind. Eng. Chem. anal. Ed., 4, 202-204.

R.R.I., Rowett Research Institute, 1978. Feedingstuffs Evaluation Unit. Second Report, 36 p.

R.R.I., Rowett Research Institute, 1981. Compound feedingstuffs for ruminants. Feedingstuffs Evaluation Unit. Third report, $49 \mathrm{p}$.

Salo M.L., 1957. Lignin studies. I. Investigations concerning lignin determination. J. Sci. Agric. Soc. Finland, 29, 185-193.

SAlo M.L., 1958. Lignin studies. III. Lignin as a tracer in digestibility investigations. J. Sci. Agr. Soc. Finland, 30, 97-104.

Salo M.L., 1965. Suomen Meataloustreteellisen Seuran Julka, 105, Acta Agralia Fennica, $1-102$.

SaRKANEN K.V.. LUdWIG C.H., 1971. Lignins : occurrence, formation, structure and reaction, 916 p. Wiley - Intersc. éd.

Sauvant D., 1981. Prévision de la valeur énergétique des aliments concentrés et composés pour les ruminants, 237-258. In «Prévision de la valeur nutritive des aliments des ruminants». Ed. I.N.R.A. Publications, Route de Saint-Cyr, 78000 Versailles. 
Sauvant D., Dorleans M., Derrien A., Giger S., 1981. Compte rendu des résultats de l'enquête I.T.E.B. sur la qualité des aliments concentrés pour vaches laitières (données non publiées).

Scales G.H., Streeter C.L., Denham A.H., Ward G.H., 1974. A comparison of indirect methods of predicting in vivo digestibility of grazed forages. J. Anim. Sci., 38, 192-199.

Schorger H., 1917. J. Ind. Eng. Chem., 9, 556 cité par Hawley L.F. et Wise L.E., 1931.

SChramm S., Bergner H., 1969. Untersuchungen zu einem neuen Verfahren der indirekten kalorimetrischen Bestimmung der Lignin in Futtermitteln. 2. Nachweis der allgemeinen Andwendbarkeit und Reproduzierbarkeit der Verfahren an verschiedenen Futtermitteln. Arch. Tierenühr., 19, 281-288.

Schulze F., 1857. Beitrag zur Kenntnis des Lignins. Chem. Zentr. B 1. 2, 321-325, cité par FISCHER, $1961 \mathrm{~b}$.

Shenk J.Q., Westerhaus M.O., Hoover M.R., 1979. Analysis of forages by infrared reflectance. J. Dairy Sci., 62, 807-812.

Sherrard E.C., Harris E.E., 1932. Ind. Eng. Chem. Anal. Ed., 24, 103, cité par Moon et ABOU RAYA, 1952 a.

SHRIKANDE J.G., 1940. Estimation of lignin in tannin materials. Biochem. J., 34, 783-789.

Southgate D.A.T., 1969. Determination of carbohydrate in foods. II. Unavailable carbohydrates. J. Sci. Food. Agric., 20, 331-335.

Southgate D.A.T., Bailey B., Collinson E., Walker A., 1976. A guide to calculating intakes of dietary fiber. J. Human nutr., 30, 303-313.

Sipinger U., 1949. Methodenbuch Bd I, S 48, Verlag Neumann, Radebeul.

STREeter C.L., 1969. A review of techniques used to estimate the in vivo digestibility of grazed forage. J. Anim. Sci., 29, 757-768.

Sullivan J.T., 1955. Cellulose and lignin in forage grasses and their digestibility coefficients. J. Anim. Sci., 14, 710-717.

Sullivan J.T., 1959. A rapid method for the determination of acid-insoluble lignin in forages and its relation to digestibility. J. Anim. Sci., 18, 1292-1298.

Sullivan J.T., 1964. The chemical composition of forages in relation to digestibility by ruminants. U.S.D.A. ARS 34-62.

Swift R.W., Thacker E.J., Black A., Bratzler J.W., James W.H., 1947. Digestibility of rations for ruminants as affected by proportions of nutrients. J. Anim. Sci., 6, 432-444.

Szentmihalyi A., 1972. Eine neue Ligninbestimmungsmethode. Sitzungsberichte, Deutsche Akademie der Landwirtschaftswissenschaften, 19, 103-105.

Thivend P., 1981. Les constituants glucidiques des aliments concentrés et sous-produits. In «Prévision de la valeur nutritive des aliments des ruminants», 219-235. Ed. I.N.R.A. Publications, Route de St-Cyr, 78000 Versailles.

Thomas B., Armstrong D.G., 1949. A study of some methods at present used for the determination of lignin. J. Agric. Sci., 39, 335-346.

Thonney M.L., Duhaime D.J., Moe P.E., Reid J.T., 1979. Acid insoluble ash and permanganate lignin as indicators to determine digestibility of cattle rations. J. Anim. Sci., 49, $1112-1116$.

Tollier M.Th., Riquet A.M., 1980. Dosage des polyosides. In «Les polymères végétaux», 155-175, éd. Gauthier-Villars, Paris.

TORP J., 1980. Variation in the concentration of major carbohydrates in the gain of some spring barleys. J. Sci. Food. Agric., 31, 1354-1360.

VAN Soest P.J., 1963. Use of detergents in the analysis of fibrous feeds. II. A rapid method for the determination of fibre and lignin. J. Assoc. Off. Anal. Chem., 46, 829-835.

VAN SOEST P.J., 1964. Symposium of nutrition and forage and pastures : new chemical procedures for evaluating forages. J. Anim. Sci., 23, 838-845.

VAN Soest P.J., 1965. Use of detergents in analysis of fibrous feeds. III. Study of effects of heating and drying on yield of fiber and lignin in forages. J. Assoc. Off. Anal. Chem., 48, 785-790. 
VAN Soest P.J., 1969. Chemical properties of fiber in concentrate feedstuffs. Proc. Cornell Nutr. Conf., 1969, 17-21.

VAN SoEst P.J., Wine R.H., 1967. Use of detergents in the analysis of fibrous feeds. IV. Determination of plant cell-wall constituents. J. Assoc. Off. Anal. Chem., 50, 50-55.

VAN Soest P.J., Wine R.H., 1968. The determination of lignin and cellulose in acid-detergent fibre with permanganate. J. Assoc. Off. Anal. Chem., 51, 780-785.

VAN Soest P.J. Robertson J.B., 1979. The detergent system of analysis and its application to human foods. E.E.C. Dietary Fiber Group, Ottawa, Canada, March, 12-14 1979.

Whitehead D.L., QUicke G.V., 1964. A comparison of six methods of estimating lignin in grass hay. J. Sci Food Agric., 15, 417-422.

Willstatter, Zechtmeister, 1913. Ber. Chem. Ges., 46, 4201. Cité par Hawley L.F. et WISE L.E. (1931).

Winch J.E., MaJor H., 1981. Predicting nitrogen and digestibility of forages using near infrared reflectance photometry. Can. J. Plant. Science, 61, 45-51.

Yu Y., 1976. Relationship between measurements of heating and acid-detergent insoluble nitrogen in heat damaged fresh alfalfa, haylage and hay. J. Dairy Sci., 59, 1845-1849. 